Meike Meliss / Christine Möhrs / Maria Ribeiro Silveira

\title{
Anforderungen und Erwartungen an eine lexikografische Ressource des gesprochenen Deutsch aus der L2-Lernerperspektive
}

$1 \quad$ Einleitung

2 Hintergrund - lexikografische Kodifizierung - Methoden

2.1 Projekthintergrund

2.2 Kodifizierung der gesprochenen Lexik in gängigen WB der deutschen Gegenwartssprache

2.3 Empirische Methoden

3 Datengrundlage und Auswertungen

3.1 LeGeDe-Befragungen: Allgemeine Information
3.2 Soziodemografische Daten und Lernerperspektive

3.3 Ausgewählte Ergebnisse

4 Fazit

5 Projektwebseite und Fragebögen

6 Literatur

6.1 Monografien und Aufsätze

6.2 Ressourcen und Online-Wörterbücher

\begin{abstract}
In the project LeGeDe (,Lexik des gesprochenen Deutsch”), we are developing a corpus-based lexicographical resource focusing on features of the lexicon of spoken German. To investigate the expectations of future users, two studies were conducted: interviews with a smaller group of experts and a large-scale online survey. We report on selected results, mainly from the online survey and with a focus on the learning perspective. We want to show if and to which extent the L2-learners' expectations differ from those of native speakers and in which aspects the two groups agree. We also want to give an outlook on the possibilities that will be available to learners in the planned lexicographical resource.
\end{abstract}

Keywords: lexicon of spoken German, digital lexicographical resource, surveys, L2-learner perspective

Schlagwörter: Lexik des gesprochenen Deutsch, Internetlexikografie, Umfragen, L2-Lernerperspektive

Meike Meliss, Universidad de Santiago de Compostela und Institut für Deutsche Sprache, Postfach 1016 21, 68016 Mannheim, e-Mail: meliss@ids-mannheim.de / meike.meliss@usc.es Christine Möhrs, Institut für Deutsche Sprache, Postfach 1016 21, 68016 Mannheim, e-Mail: moehrs@ids-mannheim.de Maria Ribeiro Silveira, Institut für Deutsche Sprache, Postfach 1016 21, 68016 Mannheim, e-Mail: ribeiro@swhk.ids-mannheim.de 


\section{Einleitung}

Das Forschungsprojekt „Lexik des gesprochenen Deutsch“ (= LeGeDe) ${ }^{1}$ strebt die Entwicklung einer korpusbasierten lexikografischen Ressource zu Besonderheiten der Lexik des gesprochenen Deutsch in der Interaktion an. Die Konzeption und Erstellung einer jeden lexikografischen Ressource erfordert eine Reihe unterschiedlicher Abläufe, für die es gilt, geeignete methodologisch und theoretisch fundierte Vorschläge auszuarbeiten. Dieser lexikografische Prozess steht $u$. a. in Verbindung mit Fragen zu (i) der anvisierten Benutzergruppe und der Benutzungssituation, (ii) der Entwicklung der Makro-, Mikro- und der Mediostruktur, (iii) den Zugriffsmöglichkeiten und letztlich (iv) der Interface-Entwicklung. Zur Unterstützung dieses Prozesses können in unterschiedlichen Projektphasen empirische Wörterbucherwartungs- und -benutzungsstudien durchgeführt werden, die je nach Zeitpunkt und Arbeitsphase des Projektes unterschiedliche Ziele verfolgen (vgl. u. a. die Arbeiten von Müller-Spitzer/Wolfer/Koplenig 2015; Müller-Spitzer 2016a oder die Studien in Müller-Spitzer (Hrsg.) 2014b).

Um die zukünftige LeGeDe-Ressource mit den Anforderungen und Erwartungen potentieller Nutzer ${ }^{2}$ abzustimmen, wurden gleich in der Projektanfangsphase (zwischen Februar und Mai 2017) Erwartungen an ein „Wörterbuch ${ }^{3}$ des gesprochenen Deutsch“ zunächst durch zwei Studien (Experteninterviews und OnlineUmfrage $^{4}$ ) empirisch erhoben und die Ergebnisse ausgewertet (vgl. die Darstellung in Meliss/Möhrs/Ribeiro Silveira 20185). Die Umfrageergebnisse zeigen u.a., dass die geplante Ressource sowohl für ein wissenschaftlich interessiertes Publikum als auch für Nutzer aus der L2-Lernerperspektive von Interesse sein könnte. Dies steht im Einklang mit der allgemeinen Beobachtung, dass der Informationsbedarf zu typisch gesprochensprachlicher Lexik auf allen Ebenen in unterschiedlichen Anwendungsbereichen steigt. Das Interesse an der Erstellung von geeigneten (Unterrichts-)Materialien wächst dabei sowohl für den Unterricht und die Lehre - speziell für den Sekundarbereich (vgl. z. B. den Beitrag von Osterroth 2018) und die Bereiche Deutsch als Fremd- und/oder Zweitsprache - sowie für das Verlagswesen (vgl.

1 Genauere Informationen zum LeGeDe-Projekt gibt es auf der Projektwebseite. [Unter: <http:// www.ids-mannheim.de/lexik/lexik-des-gesprochenen-deutsch.html>; letzter Zugriff: 08.11.2018].

2 Wenn im Folgenden in Bezug auf die Studien von „Nutzer“, „Teilnehmer“ etc. gesprochen wird, so sind in dieser generischen Form weibliche wie männliche Personen gleichermaßen gemeint.

3 Im Folgenden wird der Begriff „Wörterbuch“ (mit all seinen Flexionsformen) durch die Abkürzung „WB“ ersetzt.

4 Im weiteren Textverlauf werden diese beiden Studien mit EXPI (Experteninterview) und OU (Online-Umfrage) abgekürzt. Diese Abkürzungen stehen als Platzhalter für die verschiedenen Flexionsformen der beiden Begriffe.

5 Bei dieser Publikation handelt es sich um eine allgemeine Darstellung der Ergebnisse zu beiden Studien. 
u. a. die Beiträge in Handwerker/Bäuerle/Sieberg (Hrsg.) 2016; Imo/Moraldo (Hrsg.) 2015; Moraldo/Missaglia (Hrsg.) 2013; Reeg/Gallo/Moraldo (Hrsg.) 2012 oder Sieberg 2013). Im „Gemeinsame[n] europäische[n] Referenzrahmen für Sprachen“ heißt es zu dem Beurteilungsraster zur mündlichen Kommunikation und dem Parameter „Interaktion“ für den Lernenden von Stufe C1 z. B.: „Kann aus einem ohne weiteres verfügbaren Repertoire von Diskursmitteln eine geeignete Wendung auswählen, um seine/ihre Äußerung angemessen einzuleiten, wenn er/sie das Wort ergreifen oder behalten will, oder um die eigenen Beiträge geschickt mit denen anderer Personen zu verbinden“ (Trim/North/Coste 2001: 37). Zu pragmatischen Kompetenzen heißt es für den Lernenden von Stufe B2 dann z. B.: „Kann in Gesprächen auf angemessene Weise das Wort ergreifen und dazu verschiedene, geeignete sprachliche Mittel verwenden“ (Trim/North/Coste 2001: 124). Lehrer wie Lernende würden davon ausgehen, ein solches Repertoire z. B. in den entsprechenden Lehrmaterialien für den Unterricht oder auch in geeigneten Nachschlagewerken zu finden. Untersuchungen haben gezeigt, dass dies nur eingeschränkt der Fall ist. ${ }^{6}$ Es wird deutlich, dass die Lernenden den hier nur exemplarisch aufgezeigten Ansprüchen des europäischen Referenzrahmens nur schwerlich gerecht werden können, wenn keine spezifischen Ressourcen vorliegen, in denen die genannten gesprochensprachlich typischen Phänomene der Lexik kodifiziert vorliegen.

Obwohl aus der gesamten Forschungsprojektperspektive heraus eine wissenschaftliche Nutzergruppe als anvisierte Zielgruppe der Ressource angedacht wird, sollen in diesem Beitrag ${ }^{7}$ ganz gezielt die Anforderungen und Erwartungen an eine lexikografische Ressource zur Lexik des gesprochenen Deutsch aus der Lernerperspektive von Deutsch als Fremd- bzw. Zweitsprache ${ }^{8}$ auf der Grundlage ausgewählter Ergebnisse aus den zwei in diesem Beitrag beschriebenen Studien dargestellt werden. Ziel ist es, Möglichkeiten und Grenzen für die lexikografische Umsetzung der LeGeDe-Ressource auch für diese Nutzerperspektive aufzuzeigen und zu diskutieren.

Der Beitrag gliedert sich in drei Abschnitte. Nach der Einleitung sollen im zweiten Abschnitt Hintergrundinformationen zum Projekt, zur Kodifizierung der gesprochenen Lexik in gängigen Wörterbüchern der Gegenwartssprache und zu empirischen Untersuchungen lexikografischer Fragestellungen dargestellt werden. Im dritten Abschnitt werden ausgewählte, für die Lernerperspektive besonders relevant erscheinende Ergebnisse der beiden oben genannten Studien präsentiert und

\footnotetext{
6 Erste Ergebnisse zu Lehrbuch- und Wörterbuchanalysen in Verbindung mit gesprochensprachlichen Merkmalen werden in Meliss/Möhrs (i. Dr.) vorgestellt.

7 Wir möchten uns sehr herzlich bei Sascha Wolfer für die vielen wertvollen Anmerkungen und Hinweise bedanken, die in diesen Beitrag mit eingeflossen sind.

8 In Abschnitt 3.2 wird genauer erklärt, was in diesem Beitrag unter „Lernerperspektive“ verstanden wird.
} 
diskutiert. Dabei werden neben einigen demografischen Daten in Verbindung mit der hier relevanten Lernerperspektive Beobachtungen und Annahmen zum Status und dem Umgang mit der gesprochensprachlichen Lexik in gängigen WB präsentiert. Darüber hinaus werden weitere ausgewählte Ergebnisse zu den Erwartungen der L2-Befragten zu lexikografisch relevanten Fragestellungen (u. a. zu Zielgruppe, Nachschlagehandlung etc.) und lexikografischen Umsetzungsmöglichkeiten vorgestellt. Das Fazit mit Hinweisen zu Möglichkeiten und Grenzen der Nutzung der geplanten Ressource im L2-Bereich rundet den Beitrag ab.

\section{Hintergrund - lexikografische Kodifizierung - Methoden}

Neben einleitenden Informationen zum Projekthintergrund sollen einige Beobachtungen zu der bisherigen Kodifizierung der gesprochensprachlichen Lexik der deutschen Gegenwartssprache in gängigen Wörterbüchern exemplarisch aufgezeigt werden. Die LeGeDe-Befragungen werden außerdem in den Rahmen der Wörterbuchbenutzungsforschung eingeordnet.

\subsection{Projekthintergrund}

Das LeGeDe-Projekt ist am Institut für Deutsche Sprache (IDS, Mannheim) in der Abteilung Lexik angesiedelt und arbeitet abteilungsübergreifend in Kooperation mit der Abteilung Pragmatik. Im Projekt wird das Ziel verfolgt, eine lexikografische Ressource für das gesprochene Deutsch in der Interaktion zu erstellen. Dies stellt insofern ein innovatives Vorhaben dar, als dass es eine solche lexikografische Ressource, wie sie im LeGeDe-Projekt geplant ist, bislang weder für das Deutsche noch für andere Sprachen gibt (vgl. dazu auch Abschnitt 3.3.3). ${ }^{9}$

Da sich das Projekt nicht auf entsprechende lexikografische Vorbilder zur Kodifizierung der Lexik des Gesprochenen stützen kann, müssen für den lexikografischen Prozess des geplanten Internetwörterbuches teilweise ganz neue Wege eingeschlagen werden. Neben dem Hauptziel des LeGeDe-Projekts, eine korpusbasierte lexikografische Ressource zu Besonderheiten der Lexik des gesprochenen Deutsch in der Interaktion zu entwickeln, welche als „Ausbauwörterbuch“ bzw. als „dynamisches Wörterbuch“ (Klosa/Tiberius 2016: 77) in das Online-Wortschatz-Informationssystem Deutsch (= OWID) eingebettet wird, lassen sich folgende weitere Unterziele anführen: (i) die Bestimmung der Besonderheiten und Divergenzen von mündlichem vs. schriftlichem Sprachgebrauch im lexikalischen Bereich auf verschiedenen Ebenen (Form, Inhalt/Funktion, Situation etc.), (ii) die Entwicklung neuartiger lexikografi-

9 Vgl. dazu u. a. die folgenden Projektdarstellungen: Meliss/Möhrs 2017, Möhrs/Meliss/Batinić 2017. 
scher Angabeklassen, die u. a. auf die Funktion lexikalischer Einheiten in Interaktionskontexten Bezug nehmen, (iii) die Entwicklung innovativer lexikografischer Beschreibungsformate in multimedialer Form für hochgradig kontextualisierte lexikalische Daten und (iv) die Entwicklung weiterer korpuslinguistischer Methoden und Tools zur Abfrage, Analyse und Strukturierung von automatisch generierten korpusbasierten Daten (vgl. u.a. den Beitrag von Möhrs/Meliss/Batinić 2017). Um diese Ziele zu erreichen, befasst sich das Projekt u. a. mit Forschungs- und Methodikfragen $\mathrm{zu}$ (i) der Identifizierung von interaktionstypischen, gesprochensprachlichen lexikalischen Elementen und deren spezifischen Merkmalen im Vergleich zur Lexik der geschriebenen Sprache, (ii) der Erstellung einer Stichwortliste (vgl. Meliss et al. 2018) und (iii) der Entwicklung von korpusbasierten Verfahren für die lexikalische und interaktional ausgerichtete Datenanalyse und Beschreibung auf verschiedenen Ebenen.

Grundlage der Untersuchungen ist die Lexik des gesprochenen Deutsch in der Interaktion. Dieser spezifische Gegenstandsbereich, der durch Merkmale wie „standardnah“ und „distinktiv“ definiert wird (vgl. zu der Abgrenzung von „standardnah“ bzw. „distinktiv“ Meliss/Möhrs 2017: 44), erlaubt einerseits eine Abgrenzung $\mathrm{zu}$ anderen Sprachvarietäten und verdeutlicht andererseits, dass im Zentrum des Interesses gerade die „distinktiven“, typisch gesprochensprachlichen Merkmale in der Interaktion im Vergleich zu der Lexik der geschriebenen Sprache und der medialen Mündlichkeit stehen (vgl. den Beitrag von Deppermann/Helmer 2013). Die LeGeDe-Datengrundlage beruht auf dem Forschungs- und Lehrkorpus Gesprochenes Deutsch (= FOLK) (vgl. Schmidt 2014a/b, 2016). ${ }^{10}$ Für den Vergleich mit der geschriebenen Sprache werden entsprechende Teilkorpora aus dem Deutschen Referenzkorpus (= DEREKo) genutzt (vgl. Kupietz/Schmidt 2015; Perkuhn/Keibel/ Kupietz 2012).

$\mathrm{Zu}$ einzelnen lexikalischen Phänomenbereichen, wie z. B. zum Verbalbereich liegen umfangreiche korpusbasierte Einzelstudien vor, die gute Ausgangsdaten liefern (vgl. u.a. Deppermann 2014; Deppermann/Proske/Zeschel (Hrsg.) 2017; Deppermann/Reineke 2017; Günthner 2017; Imo 2007, 2013). Zu anderen Bereichen bzw. Wortklassen, wie z. B. zu Interjektionen, betreten wir aber eher Neuland.

10 FOLK ist integriert in das Archiv für Gesprochenes Deutsch und über die Datenbank für Gesprochenes Deutsch (= DGD; vgl. Schmidt 2017) abrufbar. Es beinhaltet Gesprächsaufnahmen und Transkripte im deutschsprachigen Raum in unterschiedlichen privaten, institutionellen und öffentlichen Kontexten.

Besondere Merkmale sind (1) medial mündlich, (2) authentisch (nicht elizitiert), (3) spontan (nicht vorbereitet), (4) ,aktuell“ (Aufnahmen ab 2003, Großteil nach 2010) und (5) standardnah. FOLK ist das größte Korpus für das gesprochene Deutsch in der Interaktion, reflektiert den technischen und methodischen „State of the art“, bietet vier Annotationsebenen (cGAT-Transkript, Normalisierung, Lemmatisierung, PoS) an, beinhaltet detaillierte Metadaten zu Sprechern und Gesprächsereignissen und ermöglicht eine strukturierte Tokensuche mit Konkordanzen. In der DGDVersion 2.10 verfügt FOLK über 230 Stunden Gesprächsaufnahmen und 2,2 Mio. Tokens. 


\subsection{Kodifizierung der gesprochenen Lexik in gängigen WB der deutschen Gegenwartssprache}

Einer der Ausgangspunkte des Projekts ist die Beobachtung, dass spezifische Informationen zur Lexik des gesprochenen Deutsch in der Interaktion in lexikografischen Nachschlagewerken nur ungenügend kodifiziert sind (vgl. Meliss 2016: 195; Moon 1998: 352; Trap-Jensen 2004: 311).

Eine stichprobenhaft durchgeführte Untersuchung zum Informationsangebot verschiedener WB zu unterschiedlichen, meist typisch gesprochensprachlich identifizierten Lemmata zeigt diesbezüglich eher ernüchternde Ergebnisse, die im Folgenden dargestellt werden. Die zur exemplarischen Untersuchung herangezogenen Stichwörter wurden entsprechend den Antworten zu der Frage aus der OU „Welche Art von Stichwörtern würden Sie in einem Wörterbuch des gesprochenen Deutsch erwarten?“ (vgl. Abschnitt 3.3.1) ausgewählt und bezüglich der von den Befragten erwähnten Wünsche zum Informationsangebot (vgl. Abschnitt 3.3.2) eines WB zur gesprochenen Lexik getestet. Es wurden die Stichwörter gucken, okay und Gott betrachtet, da es sich erwiesen hat, dass sie

(i) im Vergleich zu der geschriebenen Sprache im Gesprochenen sehr häufig auftreten, ${ }^{11}$

(ii) entweder hauptsächlich/ausschließlich im Gesprochenen verwendet werden und/oder

(iii) ein „auffälliges“ Kombinationspotenzial aufweisen und somit auch dem Wunsch der Befragten nach Berücksichtigung von „mehrteiligen Lemmata“/festen Verbindungen/Formeln/Mustern gerecht werden oder/und gesprächsstrukturierende Funktionen aufweisen.

Die Analysegrundlage bilden drei der von den L2-Befragten der OU am häufigsten konsultierten einsprachigen Onlineressourcen (Duden-online, DWDS und Ponsonline: DaF) (OU: „Welches Wörterbuch oder welche Ressource würden Sie bislang für solche Zwecke [Nachschlagen von Besonderheiten der Lexik des gesprochenen Deutsch] konsultieren?“), um gerade in ihnen Information zu typisch gesprochensprachlicher Lexik nachzuschlagen (vgl. Abschnitt 3.3.3 und Abb. 3).

Die Ergebnisse lassen sich wie folgt kritisch zusammenfassen (vgl. Tab. 1):

(i) Alle drei konsultierten WB differenzieren nicht eindeutig zwischen Medialität (geschrieben vs. gesprochen), sondern kennzeichnen mit den Etiketten Register „umgangssprachlich“ und Stil „salopp“ gesprochensprachliche Phänomene.

11 Beim Vergleich zwischen FOLK und einem Teilkorpus aus DeREKo wird als Maß mit Häufigkeitsklassen gearbeitet. Dieses Maß bietet sich aufgrund der unterschiedlichen Korpusgrößen zum Vergleich an, weil es von absoluten Zahlen abstrahiert. Die Häufigkeitsklasse (HK) zeigt, um wie viel Mal häufiger das häufigste Wort als das untersuchte Wort im Korpus auftritt: das Wort mit der HK 1 ist ungefähr $1 / 2^{1}$ (doppelt) so häufig wie das Wort mit der HK 0 , das Wort mit der HK 2 ist ungefähr $1 / 2^{2}$ (ein Viertel) so häufig wie das häufigste Wort, etc. 
(ii) Auf der Form- und Bedeutungsebene bieten die WB entsprechende Informationen an, die es u. a. erlauben, Lesarten zu disambiguieren.

(iii) Interaktionsspezifische Information bieten die WB zu einzelnen Einträgen nur in sehr resümierter Form an (vgl. z. B. in Tab. 1 zu okay aus dem DWDS die sehr übergreifende Beschreibung „drückt Zustimmung aus“). Im Mittelpunkt stehen allgemeine Angaben zur Topologie (Satzanfang/Satzende), zu bestimmten kommunikativen Funktionen (Zustimmung, Ablehnung, Erleichterung etc.) sowie zu dem Äußerungsmodus (Ausruf).

(iv) Informationen $\mathrm{zu}$ kombinatorischen Besonderheiten (Kollokationen) und festen Wendungen werden aufgelistet und teilweise mit Bedeutungsinformation und rudimentären Gebrauchs- und Funktionsinformationen ergänzt.

(v) Die Informationen, die für die drei selegierten Einträge in den drei ausgewählten WB untersucht wurden, sind in ihrer qualitativen und quantitativen Ausprägung jeweils sehr unterschiedlich und verfolgen keine erkennbar einheitliche lexikografische Methode der Datengewinnung und -strukturierung.

(vi) Die Daten stammen nicht aus interaktionsspezifischen korpusbasierten Quellen. 


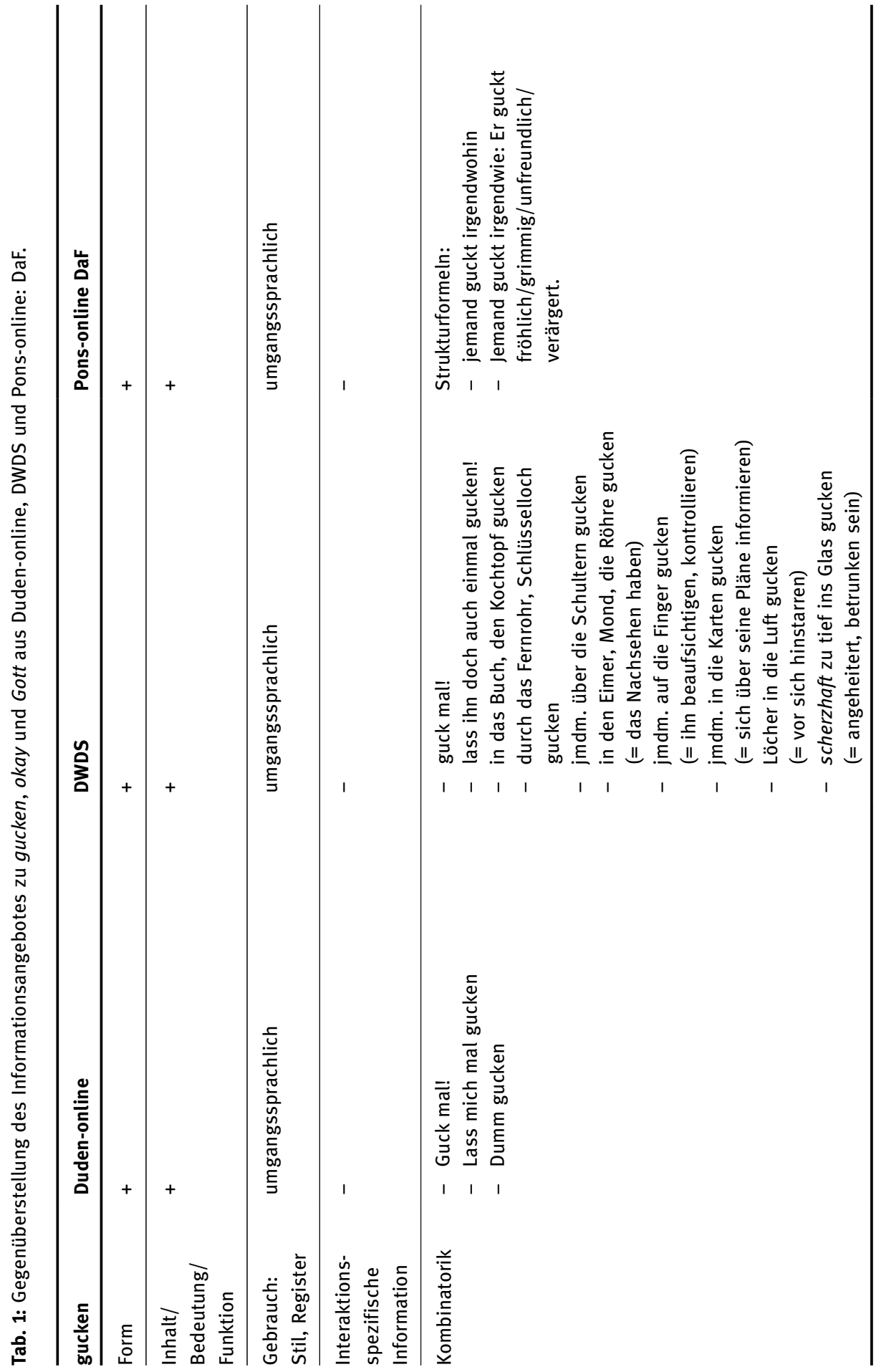




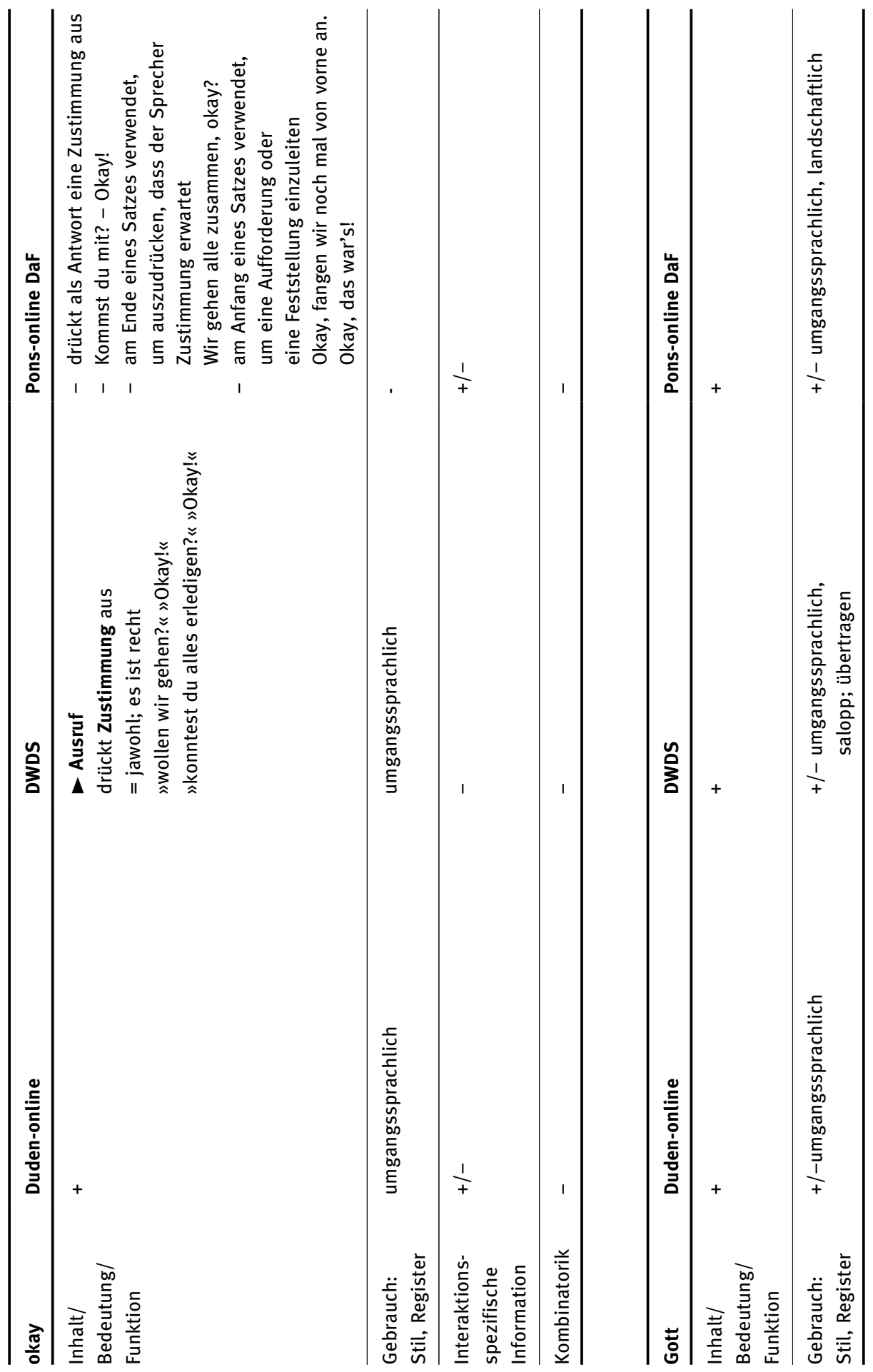




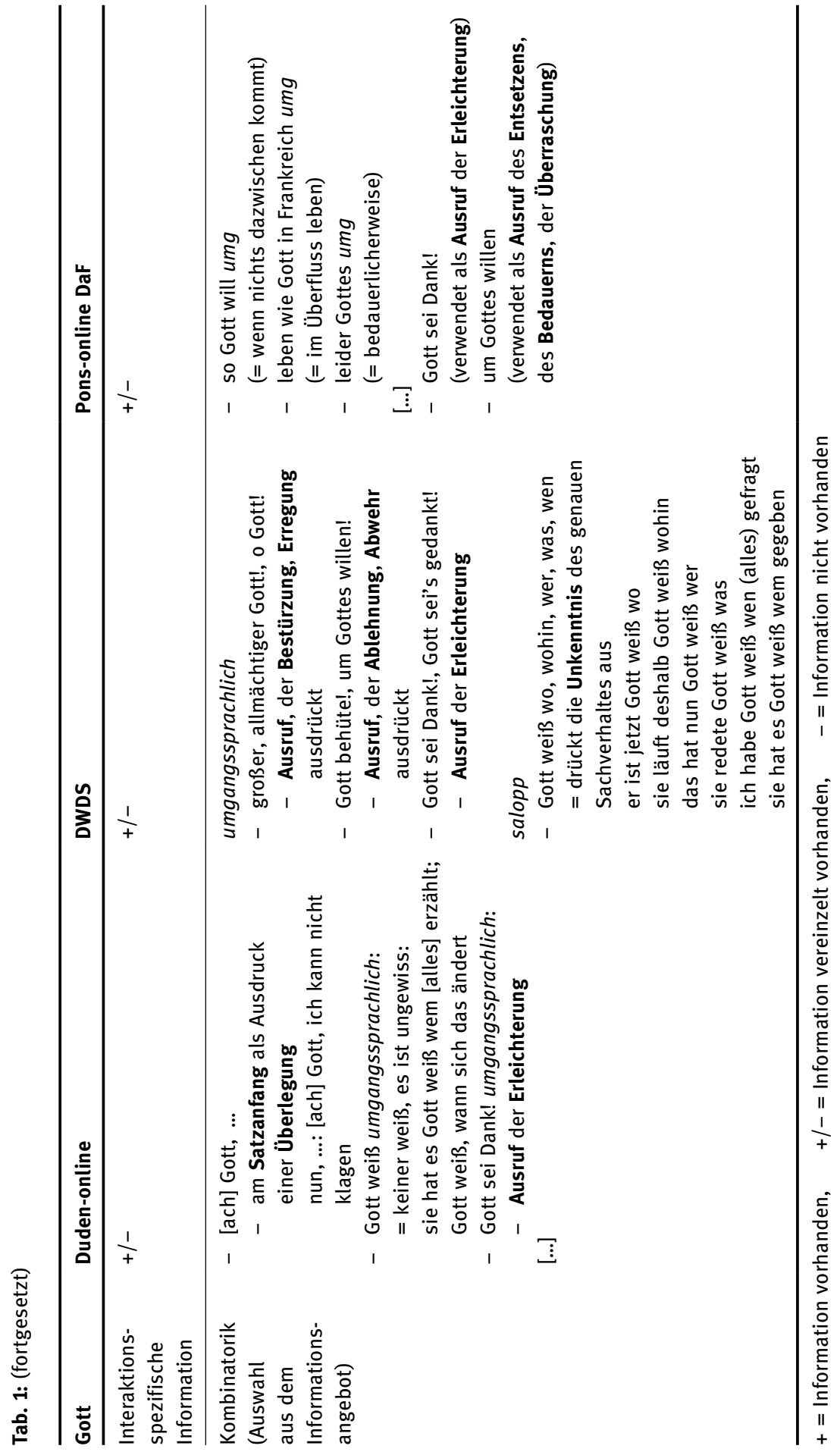




\subsection{Empirische Methoden}

Empirische Wörterbuchbenutzungsstudien können mit diversen Erhebungsmethoden und in unterschiedlichen Phasen des lexikografischen Prozesses durchgeführt werden, um verschiedene Fragestellungen zu untersuchen (vgl. u. a. die Beiträge von Bergenholtz/Johnsen 2013; Lew 2015; Müller-Spitzer 2016b; Tarp 2009; Tono 2001; Welker 2013; Wiegand 1998).

Auch im L2-Bereich kann man eine sehr rege Wörterbuchbenutzungsforschung beobachten. Es wird sogar immer wieder darauf hingewiesen, dass der Bereich der L2-Benutzungsforschung wesentlich besser untersucht sei als der der muttersprachlichen Nutzer (vgl. Bowker 2012: 380; Müller-Spitzer 2016a: 276 und 2016b: 310; Müller-Spitzer/Koplenig/Wolfer 2018: 716; Töpel 2014: 46; Welker 2010: 10). Speziell im hier besonders fokussierten DaF-Bereich lässt sich in den letzten zehn Jahren eine aktive und aktuelle Benutzungsforschung u. a. mit DaF-Lernenden romanischer Muttersprachen nachweisen (vgl. die Arbeiten von Domínguez Vázquez/ Mollica/Nied Curcio 2014; Fernández Méndez/Franco Barros/Sueiro Orallo 2016; Flinz 2014; Meliss 2015; Nied Curcio 2014 und 2015). ${ }^{12}$

Die im LeGeDe-Projekt durchgeführten Befragungen schließen an die genannten Möglichkeiten und damit an die am IDS etablierte Forschungstradition an, empirische Methoden zur Wörterbuchbenutzung auszuarbeiten (vgl. die Beiträge von Klosa/Koplenig/Töpel 2011; Müller-Spitzer/Wolfer/Koplenig 2015; Müller-Spitzer 2014a; Töpel 2014; Wolfer et al. 2014 und die Arbeiten in Müller-Spitzer (Hrsg.) 2014b). Dabei stellt das Projekt bei den Befragungen vor allem Erwartungen an die geplante Ressource in den Mittelpunkt des Erkenntnisinteresses und möchte dadurch Benutzerwünsche zu unterschiedlichen Benutzungssituationen, dem Informationsangebot, der Stichwortauswahl, den erwünschten Darstellungs- und Zugriffsmöglichkeiten etc. vor der eigentlichen Wörterbucherstellung erfahren. Die Ergebnisse der Studien zu den Erwartungen an die Ressource können dann auf den angedachten lexikografischen Prozess sowie Form und Inhalt der geplanten Ressource Einfluss nehmen und einen optimalen Zuschnitt der Ressource auf die anvisierte(n) Benutzergruppe(n) ermöglichen (vgl. Klosa/Tiberius 2016: 82).

\section{Datengrundlage und Auswertungen}

Um schon zu Beginn des lexikografischen Prozesses und der Datenmodellierung bestimmte Erwartungshaltungen zu prüfen und damit direkt auf den computerlexikografischen Prozess Einfluss nehmen zu können, wurden zwei Studien zu Erwartungshaltungen durchgeführt, die in diesem Abschnitt genauer vorgestellt werden. Die ersten beiden Abschnitte befassen sich mit allgemeinen Informationen zu den

12 Siehe dazu auch die Beiträge von Meliss et al. und von Müller-Spitzer et al. in diesem Band. 
LeGeDe-Befragungen (Abschnitt 3.1) und der genaueren Bestimmung der hier zu Grunde liegenden Lernerperspektive (Abschnitt 3.2). In Abschnitt 3.3 werden ausgewählte Ergebnisse aus den Studien vorgestellt und interpretiert.

\subsection{LeGeDe-Befragungen: Allgemeine Information}

Wie einleitend schon erwähnt, wurde zum einen ein Experteninterview (= EXPI) zum Thema „Erwartungen von Experten an die LeGeDe-Ressource“ durchgeführt, bei dem ausgewählte Fachleute in einem ca. 45-minütigen Interview befragt wurden. Zum anderen wurde auch eine breit angelegte Online-Umfrage (= OU) zum Thema „Erwartungen künftiger Nutzer an die LeGeDe-Ressource“ erstellt, die ein breiteres Teilnehmerspektrum anvisierte: Sprachwissenschaftler sowie Lehrende und Lernende der deutschen Sprache, die im universitären und außeruniversitären Kontext im Bereich Deutsch als Mutter-/Zweit- und Fremdsprache im In- und Ausland tätig sind. Die Fragebögen sind auf der Unterseite „Empirische Forschung“ der LeGeDe-Projektwebseiten einsehbar. ${ }^{13}$

Während bei den 17 ausgewählten Teilnehmern des EXPI, die zur Auswertung herangezogen wurden, hauptsächlich die qualitative Auswertung der ausführlichen Antworten auf offene Fragen von Interesse ist, steht bei den 333 Onlinefragebögen besonders die quantitative Auswertung der geschlossenen Fragen im Fokus. ${ }^{14}$

$\mathrm{Zu}$ beiden Umfragen werden Ergebnisse der deskriptiven Statistik (teilweise kombiniert mit inferenzstatistischen Verfahren) dargestellt. Beide Umfragen fokussieren inhaltlich vier verschiedene Fragenkomplexe: (i) Soziodemografische Daten, (ii) Nutzung von Online-Ressourcen allgemein, ${ }^{15}$ (iii) Einschätzungen zu der Relevanz der Spezifik der gesprochenen Lexik in unterschiedlichen Situationen und (iv) konkrete Vorstellungen, Erwartungen an eine Ressource zur gesprochenen Lexik. ${ }^{16}$ Die Auswertung der Ergebnisse wird sich im Folgenden, neben der Darstel-

13 Vgl. Unterseite „Empirische Forschung“ der Projektwebseiten [Unter: <http://www.ids-mannheim. de/lexik/lexik-des-gesprochenen-deutsch/projektbeschreibung/empirische-forschung.html>; letzter Zugriff: 08.11.2018].

14 Für genauere Informationen zur Fragentypologie verweisen wir auf Meliss/Möhrs/Ribeiro Silveira (2018) und die Projektwebseite, auf der die Fragebögen einsehbar sind.

15 Obwohl einerseits die Studie nicht primär zum Ziel hat, Daten zur allgemeinen Verwendung von (Online-)Ressourcen zu erheben, und andererseits diesbezüglich die Aussagekraft der Antworten in Frage gestellt wird (Glaubhaftigkeit des Eigenberichts), erschien es dennoch sinnvoll, einen Themenblock mit allgemeinen Fragen anzubieten. Er diente nicht nur zur Hinführung zum zentralen Thema, sondern bot andererseits auch die Chance, bestimmte Antworten bei der Interpretation zu relativieren (vgl. z. B. die Frage zur Nutzung von bestimmten Endgeräten in Abschnitt 2.3).

16 Ergebnisse zu Fragen zum Nutzungsverhalten allgemein, welches in Wörterbuchbenutzungsstudien mehrfach untersucht wurde (vgl. Abschnitt 2.2), werden aufgrund ihrer eingeschränkten Aussagekraft in Frage gestellt. Es wird darauf hingewiesen, dass der Eigenbericht der Befragten nicht immer mit dem tatsächlichen Verhalten übereinstimmt (vgl. Bergenholtz/Johnson 2005: 119; Müller-Spitzer/Koplenig/Töpel 2012: 427). 
lung einiger soziodemografischer Daten, hauptsächlich mit den Bereichen (iii) und (iv) beschäftigen.

\subsection{Soziodemografische Daten und Lernerperspektive}

Während sich im EXPI $41 \%$ der Befragten als Nichtmuttersprachler eingestuft haben („Ihre Muttersprache ist: Deutsch vs. Andere (Textfeld)“), sind es in der OU mit 97 Teilnehmenden etwas weniger als ein Drittel (L1: 70,9\% vs. L2: 29,1\%) (,Wie schätzen Sie Ihre Deutschkenntnisse ein?“). Allerdings ist $\mathrm{zu}$ beachten, dass $70 \%$ der L2-Befragten aus der OU sehr hohe Deutschkenntnisse (Selbsteinstufung im C1-C2 Bereich) aufweisen. Für die folgenden Überlegungen sollen außerdem die beruflichen Hintergründe und die Altersverteilung der L2-Befragten betrachtet werden. Dahinter steht die Hypothese, dass die Information zu diesen soziodemografischen Daten u. a. unterschiedliche Ergebnisse in Bezug auf technische - aber auch auf konzeptuelle - Erwartungen vermuten lässt, weshalb in Bezug auf weitere Überlegungen in diesem Beitrag auch immer wieder auf diese Daten zurückgegriffen wird. So sind insgesamt 42,3\% der L2-Befragten der OU in der Wissenschaft, Lehre und/oder Übersetzung tätig und können in diesem Zusammenhang als „L2-Experten“ mit einer Doppelrolle bezeichnet werden: Rolle 1 als L2-Lerner des Deutschen und Rolle 2 als Experten in Wissenschaft, Lehre und Übersetzung. In diesem Sinne sind sie „Sprachvermittler“ (Sprachwissenschaftler, DaF-/DaZ-Lehrer, Deutschlehrer (Sekundarbereich), Übersetzer). Im Gegensatz dazu können etwas mehr als die Hälfte (51,5\%) der L2-Befragten zu der Gruppe der „L2-Lerner“ (Schüler, Studierende, DaF-/DaZ-Lerner) gezählt werden (vgl. Tab. 2).

Bei Betrachtung des Alters ist auffällig, dass fast zwei Drittel der Teilnehmenden an der OU 40 Jahre oder jünger sind (63,9\%). Nimmt man nur die L2-Befragten der OU in den Blick, zeigen sich ähnliche Altersverhältnisse (L2: $\leq 40$ Jahre: 62,9\%).

Tab. 2: L2-Befragte mit ihrem beruflichen Hintergrund.

\begin{tabular}{llc}
\hline L2-„Rolle“ & Beruflicher Hintergrund der L2-Befragten & Prozent \\
\hline Experten (44,3\%) & Sprachwissenschaftler & $27,8 \%$ \\
\cline { 2 - 3 } & DaF/DaZ-Lehrer & $8,2 \%$ \\
\cline { 2 - 3 } & Deutschlehrer (Sekundarbereich) & $5,2 \%$ \\
\cline { 2 - 3 } Übersetzer & $3,1 \%$ \\
\hline Serner (51,6\%) & Studenten (Philologie) & $26,8 \%$ \\
\cline { 2 - 3 } & Deutschlerner & $22,7 \%$ \\
\cline { 2 - 3 } & Schüler & $2,1 \%$ \\
\hline
\end{tabular}


Ein genauerer Blick in die Daten verdeutlicht, dass sogar fast die Hälfte der L2Befragten der OU 30 Jahre oder jünger sind (46,4 \%). Diese Altersgruppe, die häufig als „digital natives“ 17 bezeichnet wird, ist allgemein charakterisiert durch eine hohe und ausgeprägte Medienkompetenz. Es wird allerdings auch darauf hingewiesen, dass eine allgemeine Recherchekompetenz nicht immer als selbstverständlich vorausgesetzt werden kann und dass gerade die lexikografische Nachschlagekompetenz bei L2-Lernern unzureichend sein kann (vgl. Egido Vicente/Meliss 2017: 34; Nied Curcio 2015: 446; Zöfgen 2010). In Bezug auf diese Daten können auch Ergebnisse der OU zur Nutzung von Endgeräten betrachtet werden. So zeigen die Ergebnisse, dass einige frei zugängliche Onlinedienste, wie z. B. bestimmte einsprachige und zwei- oder mehrsprachige Online-Wörterbücher ebenso wie der Google Translator, von mehr als der Hälfte aller Befragten konsultiert werden und hierfür zu ca. $60 \%$ ein mobiles Endgerät verwendet wird. Bei den jüngeren Befragten steigt die Verwendung von Notebooks für diese Zwecke auf über 70 \% (20-40 Jahre) und die von Smartphones auf fast $60 \%$ (20-30 Jahre) an. Parallel dazu erweisen sich auch das Smartphone und das Notebook als die am meisten gebrauchten Endgeräte überhaupt (vgl. Meliss/Möhrs/Ribeiro Silveira 2018: 117). Nied Curcio (2014) und Marello (2014) konnten ähnliche Beobachtungen empirisch nachweisen.

Zusammenfassend lässt sich sagen, dass die L2-Befragten der OU sowohl durch ihren beruflichen Hintergrund (Experten und Lerner) als auch durch ihre hohen Sprachkenntnisse des Deutschen gezielte und kompetenzgeleitete Erwartungen an eine Ressource des gesprochenen Deutsch sowohl aus einer theoretischen als auch aus einer praktischen bzw. anwendungsorientierten Lernerperspektive stellen können. Für die in diesem Beitrag eingenommene Lernerperspektive fließt daher die Gesamtmenge der Antworten der L2-Befragten in die Beschreibung und Interpretation der Ergebnisse ein.

Für die weiteren Überlegungen im Rahmen dieses Beitrages stehen daher hauptsächlich die Ergebnisse der L2-Perspektive der OU im Fokus. Verweise auf Ergebnisse und Erkenntnisse aus der L1-Perspektive wie auch Ergebnisse aus dem EXPI runden an einigen Stellen die Beschreibung und Interpretation ab. ${ }^{18}$ Dabei werden hauptsächlich die Auswertungen zu den Fragen von Fragenkomplex 4 (siehe Abschnitt 3.1) fokussiert und - um bestimmte Hypothesen zu prüfen - mit den soziodemografischen Daten in Verbindung gebracht.

17 Vgl. z. B. die allgemeinen Aussagen von Prensky (2001: 1) zur Beschreibung von „digital natives“: „They have spent their entire lives surrounded by and using computers, videogames, digital music players, video cams, cell phones, and all the other toys and tools of the digital age. Today's average college grads have spent fewer than 5,000 hours of their lives reading, but over 10,000 hours playing video games (not to mention 20,000 hours watching TV). Computer games, e-mail, the Internet, cell phones and instant messaging are integral parts of their lives.“

18 Da es sich beim EXPI um eine recht kleine befragte Personengruppe handelt und weitere Unterteilungen in Untergruppen nicht zu aussagekräftigen Ergebnissen führen, wird in den meisten Fällen auf die Antworten aller Befragten aus dem EXPI verwiesen. 


\subsection{Ausgewählte Ergebnisse}

\subsubsection{Bedarf und Gegenstandsbereich}

Die Ergebnisse der Antworten zu der Frage „Hätten Sie in Ihrem alltäglichen (Berufs-)Leben Bedarf für ein Wörterbuch des gesprochenen Deutsch?" aus der OU zeigen folgendes Bild: Knapp drei Viertel (74,7\%) haben auf diese Frage mit „Ja“ geantwortet. Von den 235 Befragten mit Deutsch als Muttersprache (sie werden im Folgenden als L1-Befragte bezeichnet), antworteten 72,8\% mit „Ja“. Von den $97^{19}$ Umfrageteilnehmenden mit Deutsch als Zweit- oder Fremdsprache (= L2-Befragte) weist mit 79,4\% ein leicht höherer Anteil Bedarf für ein WB des gesprochenen Deutsch im Alltag auf. Aus diesen Ergebnissen leiten sich interessante Schlussfolgerungen für den vorliegenden Beitrag zu den Anforderungen und Erwartungen an eine Ressource des gesprochenen Deutsch aus der Lernerperspektive ab.

Es existieren auf verschiedenen Ebenen Unterschiede in der Lexik des gesprochenen im Vergleich zum geschriebenen Deutsch, die dementsprechend in einer lexikografischen Ressource abrufbar sein sollten. Die Ergebnisse der Frage der OU „Welche Art von Stichwörtern würden Sie in einem Wörterbuch des gesprochenen Deutsch erwarten? “ unterstreichen diese Annahme deutlich (vgl. Abb. $1^{20}$ ). Aus den Antworten ergeben sich folgende Beobachtungen:

(i) Die meisten der Befragten der OU (87,8\%) wünschen sich in einem WB des gesprochenen Deutsch Stichwörter, die eine ,andere Bedeutung und Funktionalität“ in der gesprochenen Interaktion im Vergleich zum Geschriebenen aufweisen. Auch bei einer differenzierten Betrachtung der L1- und L2-Befragten, wurde diese Antwort von beiden Gruppen jeweils am häufigsten gewählt (gesamt: 87,8\%; L1: 89,2\% vs. L2: 84,4\%).

(ii) Drei Viertel aller Befragten der OU erwarten in einem WB des gesprochenen Deutsch sowohl Stichwörter, die als eine „Formel/usuelle Verbindung“ (gesamt: 79,5\%; L1: 81,4\% vs. L2: 75\%) einzustufen wären, als auch Stichwörter mit einem „anderen Kombinationspotenzial“ (Verbindungen/Muster, spezifische Einheiten, Kombinationspotenzial; gesamt: 74,6\%; L1: 77,1\% vs. L2: 68,8\%). Wie aus diesen Zahlen ersichtlich wird, verhalten sich die Ergebnisse der L2Befragten im Vergleich zu den L1-Befragten diesbezüglich in einem ähnlichen Verhältnis.

(iii) Stichwörter, die ,ausschließlich gesprochen“ (gesamt: 77,7\%; L1: 81\% vs. L2: 69,8\%) auftreten und solche mit besonders „häufigem Vorkommen“ in der gesprochenen Interaktion (gesamt: 71,6\%; L1: 72,3\% vs. L2: 69,8\%) werden ebenfalls von L1- und L2-Befragten der OU zu über zwei Dritteln gewünscht.

19 Die Summe der L1- und L2-Befragten ergibt 332, da ein Teilnehmer diese Frage übersprungen hat. 20 Diese Art der Darstellung bezieht sich auf Antwortoptionen mit der Möglichkeit zur Mehrfachauswahl. Da hier alle Antwortoptionen zugleich von allen gewählt werden konnten, ergibt sich für jeden einzelnen Balken ein potentieller Gesamtwert von $100 \%$. 


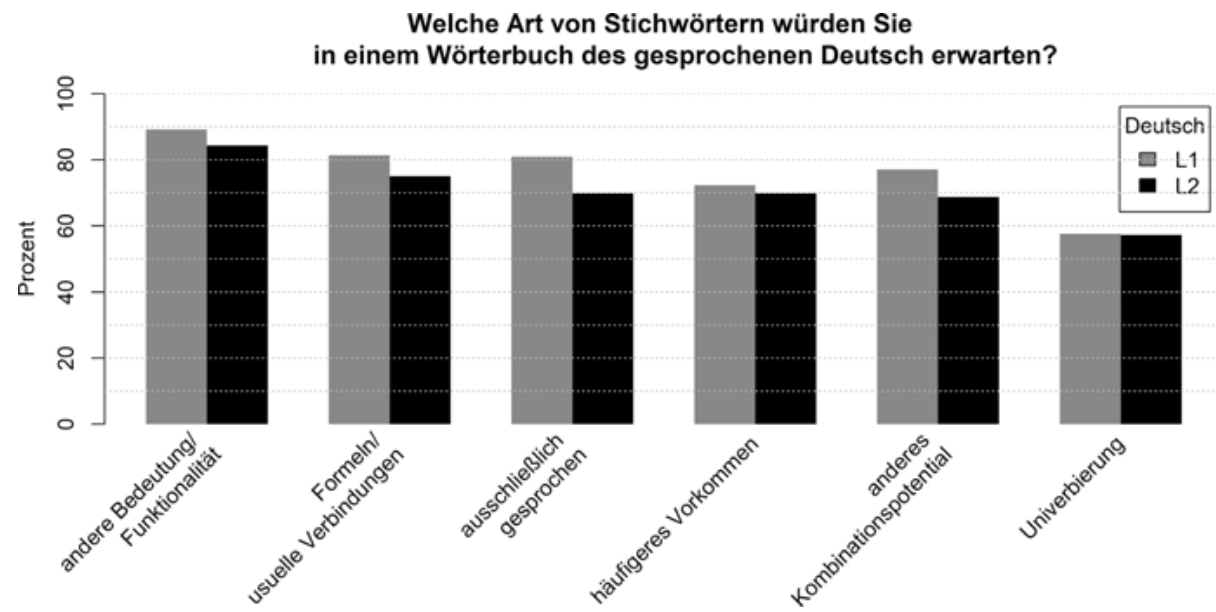

Abb. 1: Verteilung zum Gegenstandsbereich (Stichwörter) (OU).

(iv) Nur etwas mehr als die Hälfte der L1- und L2-Befragten erwarten auch entsprechende Informationen zu lexikalischen Einheiten, die sich in ihrer konkreten Ausprägung durch formal-phonetische Kontraktion bzw. „Univerbierung“ (z.B. weißte, kriegste, jein, aufm) kennzeichnen lassen (gesamt: 57,5\%; L1: $57,6 \%$ vs. L2: $57,3 \%$ ).

Bei einem genaueren Vergleich zwischen den Antworten der L1- und L2-Befragten ergeben sich zwar fast identische Rangfolgen, grundsätzlich weisen jedoch die Antworten der L2-Befragten etwas geringere Werte auf. Die Abweichungen bewegen sich auf einer Skala von ca. 4,5\% bis ca. $10 \%$. Größere Diskrepanzen innerhalb der Antworten treten bei Stichwörtern mit einem „anderen Kombinationspotenzial“ und Stichwörtern, die „ausschließlich gesprochen“ vorkommen, auf. Eine mögliche Erklärung hierfür könnte auf Prioritätsunterschiede zwischen den befragten Gruppen zurückzuführen sein. Erwartungen von Lernern (im Gegensatz zu Muttersprachlern) an ein Wörterbuch des gesprochenen Deutsch könnten auf einer grundlegenderen Ebene anzusetzen sein, die v. a. im Prozess des Spracherwerbs relevant sind.

\subsubsection{Informationsangebot}

Die im Folgenden beschriebenen Befragungsergebnisse betreffen die Behandlung der Lexik des gesprochenen Deutsch in gängigen WB. Die lexikografische Kodifizierung der typischen interaktionslinguistischen Besonderheiten der Lexik des Deutschen ist nach wie vor unzureichend. ${ }^{21}$ Diese Beobachtungen zur lexikografischen

21 Siehe dazu die Studie von Meliss (2016) zu ausgewählten Beispielen in (Lerner-)WB des Deutschen und Abschnitt 2.2 in diesem Beitrag. 
Welche Informationen sollten, Ihrer Meinung nach, in einem Onlinewörterbuch des gesprochenen Deutsch angeboten werden?

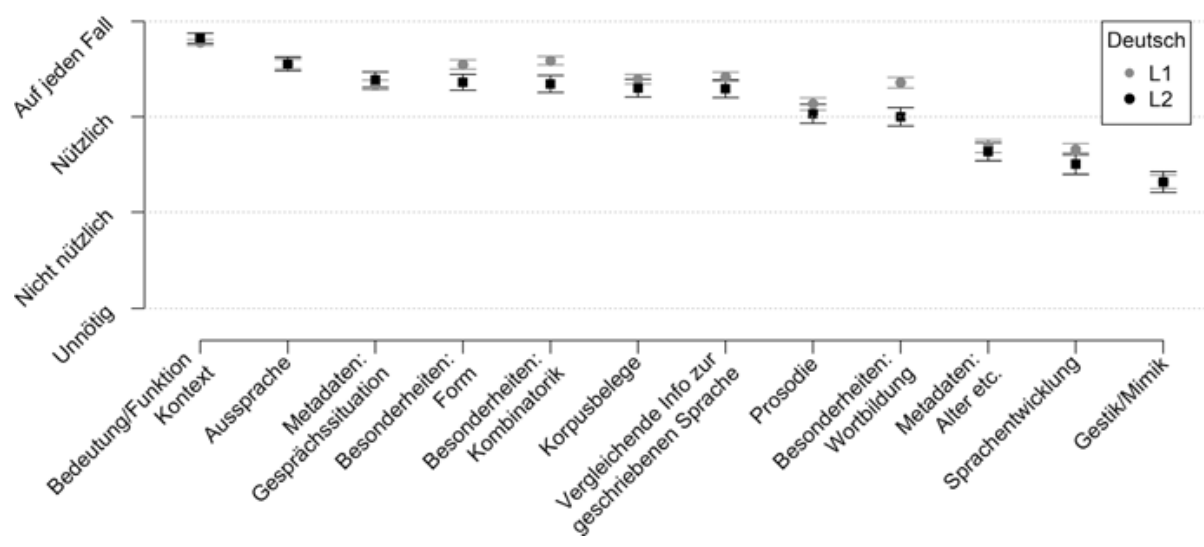

Abb. 2: Verteilung zum Informationsangebot (OU).

Kodifizierung stimmen mit den Antworten der OU auf die Frage: „Welche Informationen sollten, Ihrer Meinung nach, in einem Wörterbuch des gesprochenen Deutsch angeboten werden?“ (vgl. Abb. $2^{22}$ ) überein.

Aus den verschiedenen Antwortoptionen von „Auf jeden Fall“ bis „Unnötig“ ergibt sich eine Rangfolge, deren Mittelwerte zwischen den Stufen „Auf jeden Fall“, „Nützlich, jedoch nicht zwingend erforderlich“ und „Nicht nützlich, jedoch trotzdem wünschenswert“ rangieren. Die Ergebnisse der OU führen $\mathrm{zu}$ folgenden Beobachtungen:

(i) Die Informationen zur Aussprache, zur Bedeutung/Funktion im Kontext, zu formalen Besonderheiten sowie zu Besonderheiten in der Kombinatorik und in der Wortbildung zusammen mit dem Angebot an Korpusbelegen, Metadaten zur Gesprächssituation und vergleichender Information (geschrieben vs. gesprochen) wurden von L1- und L2-Befragten mit den Antworten „Auf jeden Fall“ bzw. „Nützlich, jedoch nicht zwingend erforderlich“ genannt.

(ii) Interessant ist weiter, dass auch Einschätzungen zwischen den Optionen „Nützlich, jedoch nicht zwingend erforderlich“ und „Nicht nützlich, aber trotzdem wünschenswert" von den L1- und L2-Befragten gleichermaßen bezüglich einiger Metadaten (Alter) und Daten zur Sprachentwicklung bewertet wurden,

22 In Abb. 2 sind die Mittelwerte (arithmetisches Mittel) der vergebenen Antworten mit Fehlerbalken (Standardfehler) dargestellt. Die Höhe der Balken gibt einen Eindruck über die Gruppengröße und die Varianz innerhalb der erhobenen Daten. Zur besseren Lesbarkeit sind die „mittleren“ Antwortoptionen „Nützlich, jedoch nicht zwingend erforderlich“ und „Nicht nützlich, jedoch trotzdem wünschenswert“ in Abb. 2 und in folgenden Abbildungen gleicher Art verkürzt dargestellt. Die Antwortoption „Ich weiß nicht“ wurde bei der Berechnung des arithmetischen Mittels nicht berücksichtigt und ist daher in den Abbildungen nicht abgetragen. 
wobei die Mittelwerte der Antworten der L2-Befragten in diesen Fällen jeweils etwas unter denen der L1-Befragten liegen.

(iii) Die Information zu Gestik/Mimik wurde von L1- und L2-Befragten jeweils mit „Nicht nützlich, jedoch trotzdem wünschenswert“ beurteilt und könnte damit als interessantes Zusatzmaterial gewertet werden.

Wenn man die Information des EXPI zum Vergleich heranzieht, dann wird deutlich, dass die Informationen zu Aussprache, Bedeutung im Kontext, Besonderheiten in der Form, Besonderheiten in der Kombinatorik, Angebot von Korpusbelegen, Metadaten zur Gesprächssituation und Prosodie von dieser Gruppe gleichermaßen mit „Auf jeden Fall“ bewertet wurden.

Ein genauerer Blick auf die L2-Daten der OU erlaubt folgende Schlussfolgerungen:

(i) Die Wünsche der OU-Befragten bezüglich des Informationsangebots für eine zukünftige Ressource der Lexik des gesprochenen Deutsch aus der L1- wie auch der L2-Sicht zeigen kaum größere Divergenzen auf. Die Werte der L2Befragten liegen allerdings immer etwas unter denen der L1-Befragten.

(ii) Eine Ausnahme bilden die Daten bezüglich der Bedeutung und Funktion im Kontext einerseits und bezüglich der Metadaten zur Gesprächssituation andererseits, für die sich im Ranking der L2-Befragten etwas höhere Werte nachweisen lassen.

(iii) Etwas weiter gehen die Einschätzungen der L1- und L2-Befragten bezüglich Besonderheiten in der Kombinatorik und in der Wortbildung auseinander: Hier ranken die L1-Befragten deutlich höher. Ein Vergleich mit den Ergebnissen zu dem Gegenstandsbereich bzw. der Frage nach der Art der Stichwörter (vgl. Abb. 1) und der Frage zum Informationsangebot (vgl. Abb. 2) erlaubt die Feststellung, dass die L1-Befragten in höherem Maße an unterschiedlich ausgeprägten Besonderheiten in Verbindung mit der Kombinatorik allgemein (anderes Kombinationspotenzial, Besonderheiten in der Kombinatorik, Wortbildung etc.) interessiert sind als die L2-Befragten (vgl. Abschnitt 3.3.1).

Die Teilnehmer des EXPI erwarten in der zukünftigen Ressource „Auf jeden Fall“ ein noch breiteres Informationsangebot als die der OU. Das zeigt sich daran, dass im EXPI fast alle Antwortoptionen zu der Frage „Welche Informationen sollten, nach Ihrer Meinung, in einem Wörterbuch des gesprochenen Deutsch angeboten werden?“ mit „Auf jeden Fall“ gewertet werden.

\subsubsection{Ressourcen}

Bis jetzt existieren kaum korpusbasierte lexikografische Projekte zur gesprochensprachlichen Lexik in der Interaktion. Modelle bzw. Vorbilder liegen für eine Ressource, wie es das LeGeDe-Projekt plant, nicht vor. ${ }^{23}$ Fast $80 \%(79,9 \%)$ der gesam-

23 Eine Ausnahme bildet das Dänische („Ordbog over Dansk Talesprog“), für das eine OnlineRessource zu Interjektionen vorliegt [Unter: <http://odt.hum.ku.dk/>; letzter Zugriff: 16.05.2018] 


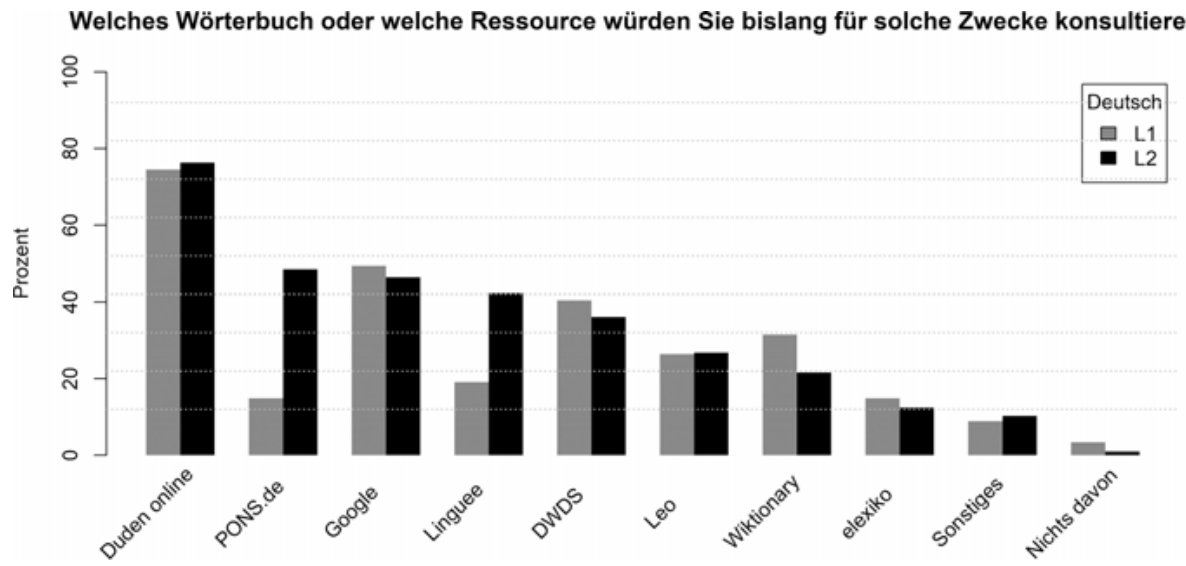

Abb. 3: Verteilung auf die bislang konsultierten Ressourcen (OU).

ten Teilnehmenden der OU geben zur Frage „Kennen Sie ein spezifisches Wörterbuch der gesprochenen Sprache und wenn ja, für welche Sprache?" auch an, dass sie kein entsprechendes WB zum gesprochenen Deutsch kennen.

Eine Aufschlüsselung der Ergebnisse nach L1- und L2-Befragten zeigt, dass die L2-Befragten noch weniger Kenntnis über mögliche existierende Ressourcen zu lexikalischen Phänomenen des Gesprochenen haben (L1: 22,03\% JA - 78 \% NEIN; L2: 15,5\%: JA - 84,5\% NEIN). Diejenigen, die angegeben haben, ein entsprechendes WB zu kennen, verweisen dann für das Deutsche hauptsächlich auf ein Aussprachewörterbuch. Diese Ergebnisse stimmen überein mit jenen aus dem EXPI. Die 17 interviewten Teilnehmer sagen aus, kein konkretes WB zum gesprochenen Deutsch zu kennen. Für andere Sprachen, wie z. B. für das Dänische, wurde vereinzelt eine entsprechende Ressource erwähnt. Ansonsten nannten die befragten Experten eher WB zur Umgangssprache und verwiesen außerdem auf lexikografische Ressourcen wie z. B. das DWDS, welches in beschränktem Umfang Informationen zur gesprochenen Sprache liefert. Andererseits wurden Korpora, die u. a. Daten zur gesprochenen Sprache anbieten, genannt.

Daran anknüpfend wurde in einer folgenden Frage im Fragebogen der OU ermittelt, welche Ressource bislang für das Nachschlagen von Besonderheiten der Lexik des gesprochenen Deutsch von den Befragten herangezogen wurde (vgl. Abb. 3).

Sehr auffällig ist, dass mit 75\% das WB Duden online mit Abstand das meistkonsultierte ist und dass die Differenzierung zwischen den Antworten der L1- und L2-Befragten diesbezüglich sogar noch höhere Ergebnisse bei den L2-Befragten aufzeigt (L1: 74,5\% vs. L2: 76,3\%). Alle anderen genannten Ressourcen wurden von

(Hansen/Hansen 2012) und ein Projekt zum Slowenischen (Verdonik/Sepesy Maučec 2017), das allerdings noch keine Ressource veröffentlicht hat. 
weniger als der Hälfte der Befragten angeführt. Dabei sind folgende Beobachtungen besonders interessant:

(i) Die zweisprachigen WB werden von L2-Befragten etwas mehr konsultiert als von L1-Befragten (PONS.de L1: 14,9\% vs. L2: 48,5\%; Linguee L1: 19,1\% vs. L2: 42,3\%; LEO L1: 26,4\% vs. L2: 28,8\%), um Besonderheiten der Lexik des gesprochenen Deutsch nachzuschlagen. Diese Daten sind im Kontext von L2Lehr- und Lernsituationen zu verstehen und stimmen mit der allgemeinen Beobachtung unterschiedlichster Benutzerumfragen überein, aus denen hervorgeht, dass L2-Lerner zweisprachige WB den einsprachigen vorziehen bzw. zweisprachige WB sehr häufig verwenden (vgl. Domínguez Vázquez/Mollica/ Nied Curcio 2014: 1; Fernández Méndez/Franco Barros/Sueiro Orallo 2016: 78; Flinz 2014: 217; Lew/Adamska-Sałaciak 2015: 50; Meliss 2015: 408; Nied Curcio 2014: 270).

(ii) Mit PONS könnte sowohl die Recherche in dem entsprechenden DaF-WB als auch in einem der zweisprachigen WB, welche über das Pons-Portal zugänglich sind, gemeint sein. Aus den Befragungsergebnissen geht dies nicht klar hervor.

(iii) Fast die Hälfte aller Befragten nennt auch Google als Ressource zum Nachschlagen von Besonderheiten der Lexik des gesprochenen Deutsch. Dieses Ergebnis wird untermauert durch die allgemeine Tendenz, Suchmaschinen für Nachschlagehandlungen heranzuziehen (vgl. Lew/de Schryver 2014: 342; Nied Curcio 2014: 270).

(iv) Wissenschaftliche WB, wie das DWDS oder elexiko, werden im Umfeld der L2Befragten weniger konsultiert. Das DWDS liegt im Unterschied zu elexiko aber noch vor LEO oder dem Wiktionary (vgl. Abb. 3). Eine mögliche Erklärung ist sicher der divergierende Bekanntheitsgrad der Ressourcen. Die seltenere Nutzung speziell dieser Ressourcen durch die L2-Befragten kann auch damit erklärt werden, dass für eine bestmögliche Nutzung höhere Deutschkenntnisse und auch lexikografische Fachkenntnisse vorhanden sein sollten (vgl. Meliss 2015: 415).

Die bisher aufgezeigten Ergebnisse verdeutlichen, dass der Bedarf an einem Wörterbuch des gesprochenen Deutsch sowohl für L1- als auch für L2-Befragte vorhanden ist. Suchstrategien führen bisher zu erläuternden Informationen in allgemeinsprachlichen Wörterbüchern oder Suchmaschinen, die (wie in Abschnitt 2.2 dargestellt) als unzureichend betrachtet werden können.

\subsubsection{Zielgruppe und Nachschlagehandlung}

Neben den allgemeinen Fragen zur Verwendung von Onlinewörterbüchern und der Einschätzung zu Bedarf und Recherchemöglichkeiten von Besonderheiten der Lexik des gesprochenen Deutsch wurden außerdem Fragen zur Zielgruppe der Ressource gestellt. Die Antworten auf die Frage der OU „Für welche Zielgruppe könnte ein Wörterbuch des gesprochenen Deutsch besonders interessant sein?" zeigen, dass 


\section{Für welche Zielgruppe könnte ein Wörterbuch des gesprochenen Deutsch besonders interessant sein?}

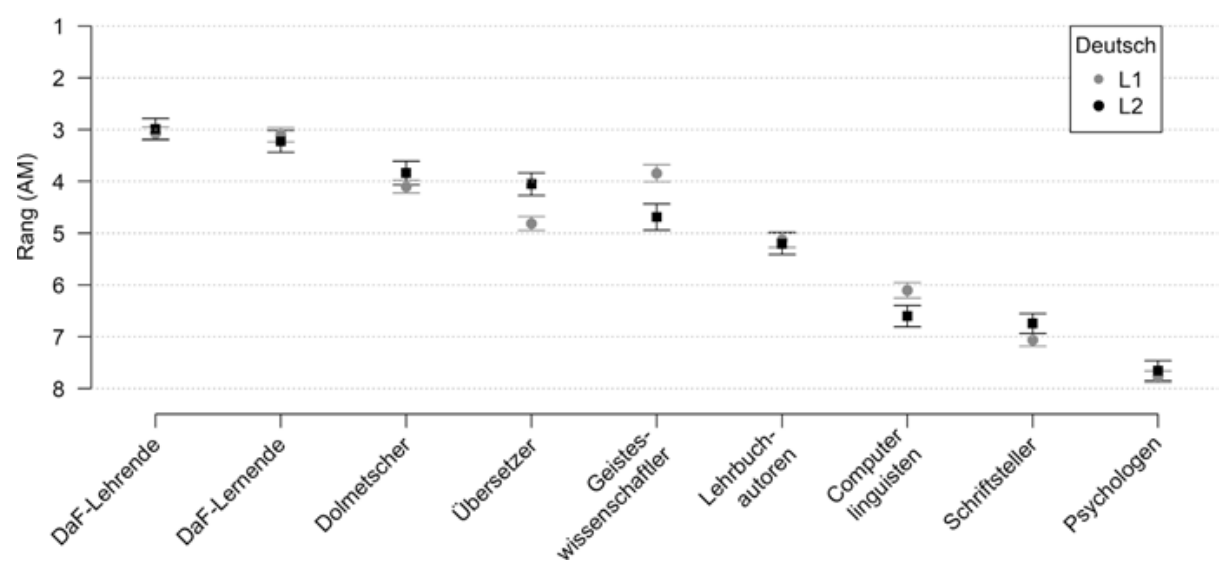

Abb. 4: Verteilung zur Frage nach der Zielgruppe (OU).

DaF/DaZ-Lehrer und -Lerner von allen L1- und L2-Befragten als Hauptzielgruppe einer lexikografischen Ressource des gesprochenen Deutsch gesehen werden (vgl. Abb. 4). Die Abweichungen zwischen den Einschätzungen der L1- und L2-Befragten diesbezüglich sind nicht auffällig. Diese Verteilung verstärkt wiederum die in Abschnitt 3.3.1 aufgestellte Überlegung zu unterschiedlichen Ansprüchen im Bereich des Fremdspracherwerbs.

Als zweitwichtigste Gruppe werden Dolmetscher und Übersetzer zusammen mit Lehrbuchautoren und Geisteswissenschaftlern genannt. Interessant dabei ist, dass es für diese zwei Gruppen zwischen den Meinungen der L1- und L2-Befragten Abweichungen gibt, die sich natürlich aus ihrem jeweiligen beruflichen Interessensumfeld ergeben. Aus der L2-Lernerperspektive gesehen sind auch die Dolmetscher und Übersetzer eine relevante Zielgruppe, während die L1-Befragten sie als etwas weniger wichtige Gruppe einstufen. L2-Befragte bewerten dagegen die Geisteswissenschaftler als weniger relevante Zielgruppe.

Im EXPI zeigen sich diesbezüglich gering verschobene Verhältnisse. Als Hauptzielgruppe werden hier an erster Stelle Wissenschaftler, direkt danach aber auch hier die DaF/DaZ-Lehrer und -Lerner gleichermaßen genannt. Die dritte Gruppe bilden die Dolmetscher, Übersetzer und Lehrbuchautoren. Als weitere mögliche Zielgruppen wurden auch u. a. folgende genannt: interessierte Öffentlichkeit, Deutschlehrer, Schüler und Germanistikstudierende. ${ }^{24}$

Durch diese Frage kann eine Verbindung zu der Frage nach möglichen Nachschlagehandlungen hergestellt werden. Die Auswertung der Antworten auf die

24 Dieses Bild ergibt sich aus der Auswertung der Antwortoption „Sonstiges“ und freien Antworten zu dieser Frage aus dem EXPI. 


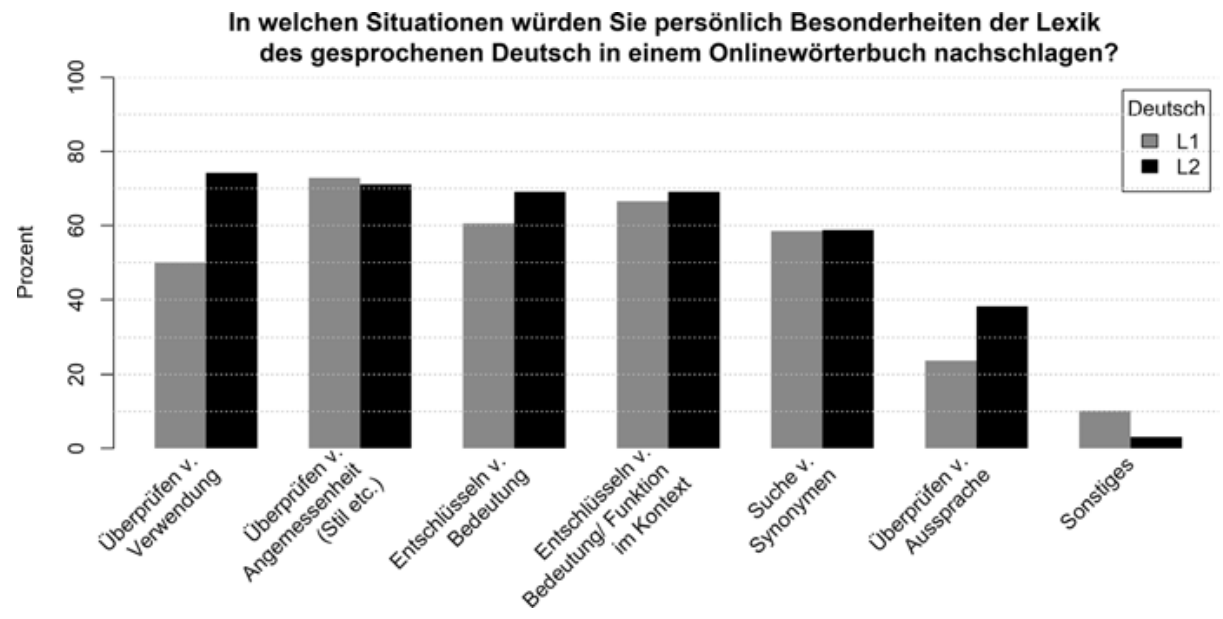

Abb. 5: Verteilung auf Nachschlagesituationen von Besonderheiten der Lexik des gesprochenen Deutsch (OU).

Frage der OU: „In welchen Situationen würden Sie persönlich Besonderheiten der Lexik des gesprochenen Deutsch in einem Onlinewörterbuch nachschlagen?" (vgl. Abb. 5) zeigt gleichermaßen Situationen der Sprachproduktion wie die der Rezeption auf (es geht um Schlagwörter wie: „überprüfen“, „suchen“, „entschlüsseln“).

Das Überprüfen von „Angemessenheit“ bezüglich Stil und Register etc. steht mit über $70 \%$ an erster Stelle der Gesamtantworten und bleibt auch bei einer differenzierten Betrachtung zwischen L1- und L2-Befragten an erster Position bei den L1-Befragten (gesamt: 72,4\%; L1: 72,9\% vs. L2: 71,1\%). Die L2-Befragten hingegen nennen das „Überprüfen von korrekter Verwendung“ an erster Stelle (gesamt: 57,1\%; L1: $50 \%$ vs. L2: 74,2\%). Dass die muttersprachlichen Befragten (L1) die „korrekte Verwendung“ im Vergleich weniger häufig nachschlagen würden als die L2-Befragten, überrascht nicht.

Das Entschlüsseln von interaktionsspezifischer „Bedeutung/Funktion im Kontext“ (gesamt: 67,3\%; L1: 66,5\% vs. L2: 69,1\%) und die allgemeine „Bedeutungsentschlüsselung“ (gesamt: 63,1\%; L1: 60,6\% vs. L2: 69,1\%) wird von allen Befragten mit zwischen $60 \%$ und $70 \%$ am zweithöchsten eingestuft. Für beide Nachschlagesituationen sind die Ergebnisse der L2-Befragten deutlich höher. Die Suche nach „Synonymen“ ist hingegen für beide Gruppen ähnlich relevant (gesamt: 58,6\%; L1: 58,5\% vs. L2: 58,8\%). Das Überprüfen von Information zur „Aussprache“ wird weit weniger - nur von etwas mehr als einem Viertel der Befragten - nachgeschlagen (gesamt: 27,9\%; L1: 23,7\% vs. L2: 38,1\%).

Im Vergleich zwischen L1- und L2-Befragten fällt auf, dass die Rangfolge bezüglich der einzelnen Nachschlagesituationen nicht ähnlich verläuft und dass alle vorgegebenen Situationen von den L2-Befragten immer jeweils höher eingestuft wurden (Ausnahme bildet nur das Überprüfen von „Angemessenheit“). Ein besonders großer 
Unterschied besteht bei den Situationen, die eher mit der „Sprachproduktion“ in Verbindung stehen: Überprüfung von „Aussprache“, Überprüfen von korrekter „Verwendung“. Dieses Ergebnis lässt sich mit der Tatsache begründen, dass L2-Sprecher automatisch einen höheren Bedarf danach haben, ihre fehlende Sprachkompetenz mit Hilfe eines Nachschlagewerkes zu überprüfen bzw. abzusichern, als dies bei L1-Sprechern der Fall ist. Die Auswertung von „Sonstigen“ Antworten beider Umfragen verweisen außerdem noch auf Forschungssituationen und Situationen im Lehr- und Lern-Kontext. ${ }^{25}$

\subsubsection{Zugriffsmöglichkeiten}

Die Frage „Welche Zugriffsmöglichkeiten halten Sie für ein Wörterbuch des gesprochenen Deutsch für besonders relevant?“ der OU wurde recht einstimmig beantwortet (vgl. Abb. 6). Alle Teilnehmer der OU halten eine alphabetische Anordnung, einen Zugriff über eine Suchmaske (erweiterte Suche), einen Zugriff über kommunikative Funktionen und über Themen- und Bedeutungsfelder für besonders relevant (zwischen „Auf jeden Fall“ und „Nützlich, jedoch nicht zwingend erforderlich“).

Auch eine innovative Zugriffsmöglichkeit über „Spracherkennung“ wurde als „Nützlich, jedoch nicht zwingend erforderlich“ angesehen. Interessant ist diesbezüglich die Tatsache, dass die L2-Befragten einen möglichen Zugriff über Spracherkennung für relevanter halten als die L1-Befragten. Da sich die fremdsprachige Rezeption in der mündlichen Interaktion als besonders komplex erweist, ist die hier aufgedeckte unterschiedliche Einschätzung der L1- und L2-Befragten gut nach-

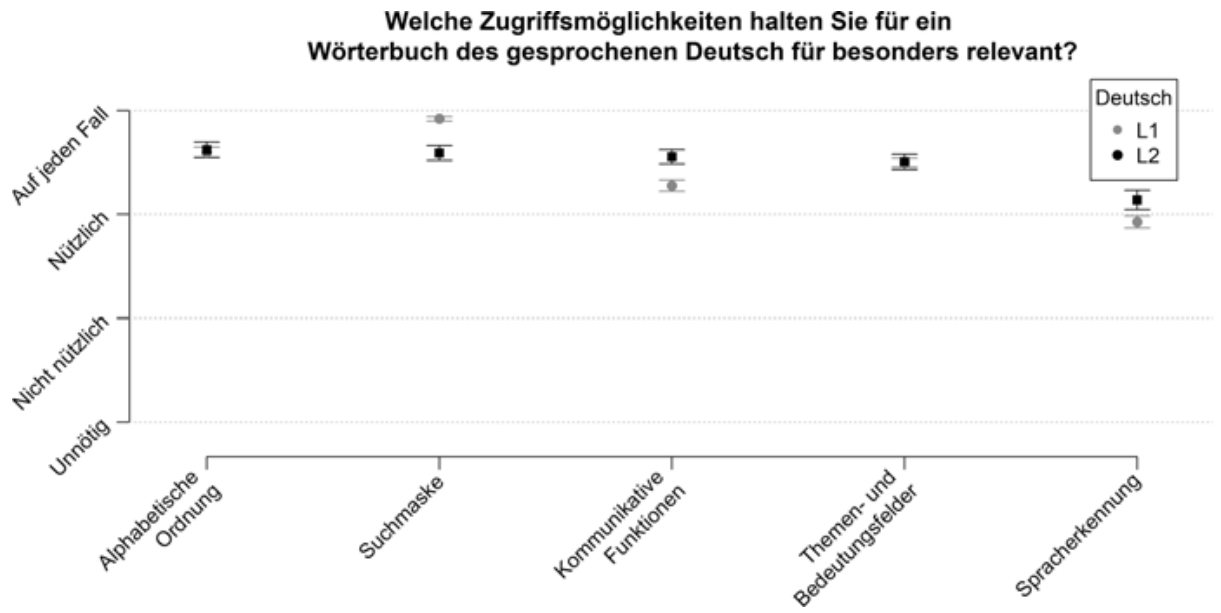

Abb. 6: Verteilung zu relevanten Zugriffsmöglichkeiten (OU).

25 Die Experten aus dem EXPI nennen außerdem Situationen wie: zur Unterrichtsvorbereitung und zu Forschungszwecken. 
zuvollziehen. Ein weiterer nennenswerter Unterschied zwischen den L1- und L2Befragten liegt in der Bewertung bezüglich der Zugriffsmöglichkeit über „Kommunikative Funktionen“ vor. Hier hätten die L2-Befragten gerne „Auf jeden Fall“ einen solchen Zugriff während die L1-Befragten diesen Zugriff etwas weniger gewichten. Eine mögliche Erklärung für diesen Unterschied steht sicher in Zusammenhang mit dem höheren Bedürfnis, in L2-Produktionssituationen für bestimmte Inhalte und Funktionen einen sprachlichen Ausdruck zu suchen.

Diese Daten zu den gewünschten Zugriffsmöglichkeiten wurden mit verschiedenen soziodemografischen Daten (Alter und beruflicher Hintergrund) abgeglichen, um der Frage nachzugehen, ob jüngere Studienteilnehmer und Experten sich „extravagantere“ Zugriffsmöglichkeiten wünschen. Aber weder das Alter noch der berufliche Hintergrund beeinflussen die hier aufgezeigten Daten in auffälliger Weise. Eine genauere Betrachtung der Ergebnisse der L2-Befragten konnte diesbezüglich ebenfalls keine divergierende Erwartungshaltung nachweisen.

\subsubsection{Verlinkungsmöglichkeiten}

Bei allen Teilnehmenden der OU sind verschiedene Arten von Verlinkungsmöglichkeiten erwünscht, welche durch die Frage „Welche Art von Verlinkungen würden Sie sich wünschen?" ermittelt wurde (vgl. Abb. 7). Dabei werden Verlinkungen zu externen akademischen WB (74,2\%) den Verlinkungen zu externen Verlags-WB (56,8 \%) vorgezogen. Eine direkte Verlinkung zu FOLK-Korpusdaten (59,5\%) wird gegenüber Verlinkungsmöglichkeiten zu anderen linguistischen Korpora (49,9\%) präferiert. Aus weiteren Fragen, die in der OU zu FOLK gestellt wurden, ${ }^{26}$ zeigt sich, dass auch Befragte, die bisher selten oder nie mit FOLK gearbeitet haben, eine Verlinkung zu dessen Korpusdaten wünschen.

Ca. zwei Drittel wünschen sich auch jeweils weitere Verlinkungsmöglichkeiten innerhalb der LeGeDe-Ressource (68,2\%) und innerhalb des Portals OWID (64,7\%).

Eine differenzierte Betrachtung der Daten der L1- und L2-Befragten zeigt eine ähnliche Rangfolge. Dabei ist auffällig, dass erneut die Antworten der L2-Befragten immer etwas unter denen der L1-Befragten liegen. Es gibt nur zwei Ausnahmen: die Antworten (i) zu Verlinkungen zu anderen Online-Ressourcen (gesamt: 30,9\%;

26 Fast alle Teilnehmer der OU wünschen sich entweder „Auf jeden Fall“ (40,8\%) oder „Eher ja“ $(40,8 \%)$ die Möglichkeit, ausgehend von einem Stichwort über Verlinkung zu einer FOLK-Recherche zu gelangen. Eine Unterscheidung zwischen L1- und L2-Befragten ergibt hier keine auffälligen Unterschiede. Die Tatsache, dass über $12 \%$ bei dieser Frage nach der Verlinkung zu FOLK mit „Ich weiß nicht" geantwortet haben, deutet allerdings darauf hin, dass FOLK nicht in allen Kreisen bekannt ist. Diese Einschätzung lässt sich auch im Zusammenhang mit den Ergebnissen auf die Frage „Haben Sie schon einmal mit FOLK (Forschungs- und Lehrkorpus Gesprochenes Deutsch) gearbeitet?“ aus der OU interpretieren: Mehr als drei Viertel der L2-Befragten haben „Nie“ mit FOLK gearbeitet. Betrachtet man dieses Ergebnis auch in Relation zu den L1-Befragten und im Verhältnis zu allen befragten Personen, so wird deutlich, dass FOLK als Ressource allen Befragten insgesamt eher unbekannt ist (Antwort „Nie“: gesamt: 70,9\%; L1: $66 \%$ vs. L2: 83,5\%). 


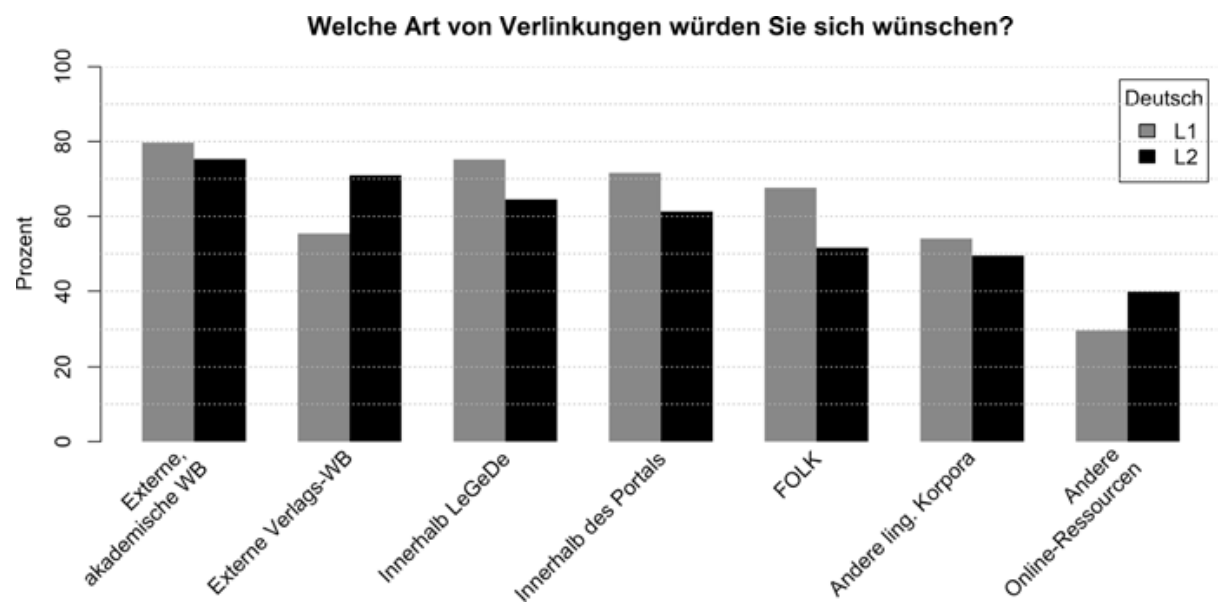

Abb. 7: Verteilung zu Verlinkungsmöglichkeiten (OU).

L1: $28 \%$ vs. L2: 38,1\%) und (ii) zu der Verlinkung zu externen Verlags-WB. In diesem Fall liegen die Antworten der L2-Befragten um über $10 \%$ höher als die der L1Befragten (gesamt: 56,8\%; L1: 52,1\% vs. L2: 68\%). Eine Erklärung liegt eventuell darin, dass die L2-Befragten neben der häufigen Konsultation von freien lexikografischen Verlagsressourcen auch andere Onlinedienste im stärkeren Maße als die L1Befragten nutzen (was in der OU über „Welche (frei zugänglichen) Onlinedienste verwenden Sie regelmäßig?““ abgefragt wurde).

\subsubsection{Präsentationsmodalitäten und innovative Funktionalitäten}

Auf die Frage, welche Präsentationsmodalitäten die neue Ressource ausnutzen sollte wurde von allen Teilnehmenden der OU „Auf jeden Fall“ Audio auf Platz 1 gerankt. ${ }^{27}$ Zwischen Platz 2 und 3 wurde ohne größere Unterschiede zwischen L1- und L2-Befragten die Information zu folgenden Modalitäten als „Nützlich, jedoch nicht zwingend erforderlich“ angesehen: Graphiken, Tabellen, Transkripte, Video und Wortwolken. Auf Platz 3 („Nicht nützlich, jedoch trotzdem wünschenswert“) wurden Oszillogramme eingestuft (vgl. Abb. 8). ${ }^{28}$

Größere Unterschiede zwischen den Einstufungen der L1- und L2-Befragten liegen hauptsächlich bei Transkripten in GAT. Je fachspezifischer die Präsentationsmodalitäten sind, desto weniger nützlich stufen die Befragten sie für die geplante Ressource ein. Besonders auffällig ist der eindeutige Wunsch sowohl der L1- als

27 Auch in der EXPI haben die Interviewten bei dieser Frage („Welche Präsentationsmodalitäten und Verweisoptionen sollte das Wörterbuch ausnutzen?“) „Audio“ als wichtigste Präsentationsmodalität vor Transkripten und Videos genannt.

$28 \mathrm{Zu}$ den innovativen Möglichkeiten haben die Befragten der EXPI ähnliche Wünsche wie die der OU. 
Welche Präsentationsmodalitäten sollte das Wörterbuch ausnutzen?

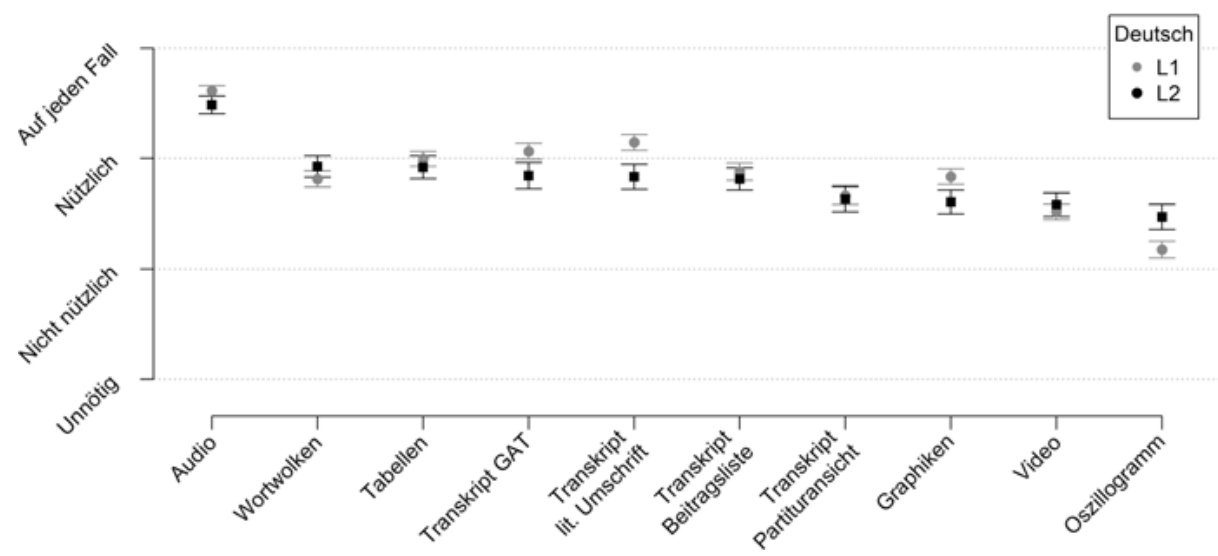

Abb. 8: Verteilung zu Präsentationsmodalitäten (OU).

auch der L2-Befragten, „Auf jeden Fall“ ein Angebot von Audiodateien als eine mögliche Präsentationsmodalität zu erhalten. Die Antworten verdeutlichen den Wunsch aller Befragten, „Auf jeden Fall“ Informationen zur Aussprache zur Verfügung gestellt zu bekommen (vgl. Abschnitt 3.3.2, Abb. 2). Dass das Überprüfen von Aussprache als Nachschlagehandlung von den Befragten auf die Frage „In welchen Situationen würden Sie persönlich Besonderheiten der Lexik des gesprochenen Deutsch in einem Onlinewörterbuch nachschlagen?" im Vergleich $\mathrm{zu}$ anderen Nachschlagehandlungen eher wenig genannt wurde (vgl. Abschnitt 3.3.4, Abb. 5), könnte einerseits darauf hinweisen, dass die Befragten mit dem bisherigen Audioangebot der von ihnen häufig konsultierten lexikografischen Ressourcen nicht zufrieden sind und dieses Informationsangebot daher weniger nutzen als andere (vgl. Abschnitt 3.3.3, Abb. 3). Andererseits kann man aber auch diese Daten mit dem besonders von den L2-Befragten geäußerten Wunsch nach einem Zugriff über Spracherkennung in Verbindung setzen (vgl. Abschnitt 3.3.5, Abb. 6).

Ein Abgleich der Daten mit dem Alter zeigt, dass die Antworten aller Teilnehmenden der OU der Altersstufe 40-50 bei fast allen Werten etwas tiefer als der Mittelwert liegen (Graphiken, Oszillogramm, Transkript Beitragsliste, Transkript GAT) und sich bei Bewertungen zwischen „Nicht nützlich, jedoch trotzdem wünschenswert“ und „Unnötig“ bewegen. Bezüglich weiterer Wünsche nach innovativen Funktionalitäten haben die Auswertungen der OU ergeben, dass neben den schon erwähnten Möglichkeiten auch u.a. noch folgende erwünscht sind: Spracherkennung (7,3\%), Dialog mit Nutzern/Team (3,3\%), Nutzerbeteiligung (3,3\%), Zugriff über IPA (3,3\%). 


\section{Fazit}

Aus den Ergebnissen der durchgeführten Online-Befragung und des Experteninterviews, mit denen die Erwartungen zukünftiger Nachschlagender an die LeGeDeRessourcen untersucht wurden, lassen sich zu den Anforderungen und Erwartungen insbesondere aus der Lernerperspektive folgende Ergebnisse zusammenfassen.

Nur sehr wenige Befragte kennen eine lexikografische Ressource, die sich speziell mit Besonderheiten der gesprochenen Lexik auseinandersetzt (vgl. Abschnitt 3.3.3). Die Ressourcen, die bisher von den L2-Befragten hauptsächlich für das Nachschlagen von Besonderheiten der Lexik des gesprochenen Deutsch verwendet wurden, bieten kaum und in nur sehr unzureichender Form Information $\mathrm{zu}$ lexikalischen und interaktionsspezifischen Besonderheiten an, wie auch in dem Abschnitt zur Kodifizierung der gesprochenen Lexik in gängigen WB der deutschen Gegenwartssprache exemplarisch aufgezeigt werden konnte. Unsere Annahmen über die Kodifizierung von interaktionsspezifischen Informationen in bislang von den Befragten konsultierten Wörterbüchern und über den Bedarf an geeigneten Nachschlagewerken zur Lexik des gesprochenen Deutsch in der Interaktion (vgl. Abschnitt 2.2) konnten durch die aufgezeigten Ergebnisse bestätigt werden und zeigen sowohl für L1- als auch für L2-Befragte den Wunsch und die Notwendigkeit auf, für bestimmte Nachschlagehandlungen eine spezifische Ressource zum gesprochenen Deutsch konsultieren zu können. Diese Beobachtungen passen zu den Anforderungen im Rahmen der Entwicklung der mündlichen Kompetenz, wie sie im „Gemeinsame[n] europäische[n] Referenzrahmen für Sprachen“ gefordert wird (vgl. Abschnitt 1). So stehen DaF-Lehrende und -Lernende - nach Einschätzung aller befragten Personen - als Zielgruppe an erster Stelle (vgl. Abschnitt 3.3.4). Es überrascht daher nicht, dass gerade für Nachschlagesituationen in Verbindung mit Produktionssituationen die L2-Befragten einen hohen Bedarf angegeben haben (vgl. Abschnitt 3.3.4). Gleiches gilt für die Fragen nach dem gewünschten Gegenstandsbereich und dem Informationsbedarf. Die erhobenen Daten zu den Erwartungen in Bezug auf die Stichwörter in einem Wörterbuch des gesprochenen Deutsch wiesen bezüglich der vorgegebenen möglichen Mehrfachantworten zwar jeweils etwas niedrigere Werte für die L2-Befragten (im Vergleich zu den L1-Befragten) auf, aber in Bezug auf die erwarteten Stichwörter in der geplanten Ressource kann dennoch festgestellt werden, dass sich aus den Daten keine größere Diskrepanz bezüglich der Erwartungshaltung beider Gruppen schließen lässt (vgl. Abschnitt 3.3.1). Die Wünsche der L1- und L2-Befragten zum Informationsangebot ähneln sich im Ranking ebenfalls. Die etwas höheren Mittelwerte in den Rankings der L2-Befragten in Verbindung mit dem Wunsch, „Auf jeden Fall“ Information zu Bedeutung und Funktion im Kontext und den Informationen zu Metadaten der Gesprächssituation zu erhalten, lassen sich sicher damit erklären, dass L2-Lerner allgemein mehr Kontextinformation für die fremdsprachliche Rezeption und Produktion benötigen (vgl. Abschnitt 3.3.2). Die Tatsache, dass die L2-Befragten eine 
Zugriffsmöglichkeit über „Kommunikative Funktionen“ höher gewichten als die L1Befragten, kann auf einen höheren Bedarf an Nachschlagehandlungen für Produktionszwecke zurückgeführt werden (vgl. Abschnitte 3.3.4 und 3.3.5). Die Wünsche zu möglichen Verlinkungen auf andere externe Ressourcen und Korpora ist bei den L2-Befragten stark abhängig von ihrem beruflichen Hintergrund und Kenntnisstand. Besonders auffällig ist, dass die L2-Befragten die externen Verlinkungsmöglichkeiten zu Verlags-WB in hohem Maße anführen, im Gegensatz dazu aber eine Verlinkung zu FOLK weniger wünschen als die L1-Befragten, was damit erklärt werden konnte, dass im L2-Bereich dieses Korpus kaum bekannt und folglich kaum genutzt wird (vgl. Abschnitt 3.3.6). Schließlich wünschen sich sowohl die L1- als auch die L2-Befragten gleichermaßen die unterschiedlichsten Präsentationsmodalitäten (vgl. Abschnitt 3.3.7) und innovative Funktionalitäten. Neben dem Wunsch nach Audiodateien, die von allen befragten Personen als die wichtigste Präsentationsmodalität genannt wurde, wird aber deutlich, dass die L2-Befragten mehr als die L1-Befragten das Bedürfnis nach einfacheren Präsentationsformen anmelden (z. B. Tabellen, Wortwolken).

Die geplante LeGeDe-Ressource soll zunächst als Wissensspeicher und Wortschatzdokumentation fungieren und Wissenschaftler, Gesprächsforscher und Lexikologen als primäre Zielgruppe anvisieren. Dennoch erlaubt der hier eingenommene Blick aus der Lernerperspektive auf die erhobenen Daten das Fazit, dass auch DaF-Lerner in allen Stufen von der geplanten Ressource profitieren können, wenn die Experten (Wissenschaftler, Lehrer etc.) die entsprechende Mittlerposition einnehmen. Dies könnte zum Beispiel durch eine adäquate bzw. benutzeradaptierte Metasprache oder eine entsprechend angepasste Informationsauswahl erfolgen, um auch Lehrern und Lehrbuchautoren die Möglichkeit einzuräumen, auf der Informationsbasis der geplanten Ressource entsprechende Lehr- und Lernmaterialien zu entwickeln.

\section{Projektwebseite und Fragebögen}

LeGeDe Projektwebseite. [Unter: <http://www.ids-mannheim.de/lexik/lexik-des-gesprochenendeutsch.html>; letzter Zugriff: 08.11. 2018].

Die Fragebögen sind auf der Unterseite „Empirische Forschung“ der Projektwebseite einsehbar. [Unter: <http://www.ids-mannheim.de/lexik/lexik-des-gesprochenen-deutsch/ projektbeschreibung/empirische-forschung.html>; letzter Zugriff: 08.11. 2018].

\section{Literatur}

\subsection{Monografien und Aufsätze}

Bergenholtz, Henning/Johnsen, Mia (2005): Log files as a tool for improving internet dictionaries. In: Hermes 34, 117-141. 
Bergenholtz, Henning/Johnsen, Mia (2013): User research in the field of electronic dictionaries: Methods, first results, proposals. In: Gouws, Rufus H. et al. (Hrsg.): Dictionaries. An International Encyclopedia of Lexicography. Supplementary Volume: Recent Developments with Focus on Electronic and Computational Lexicography. Berlin/Boston: de Gruyter, 556-568.

Bowker, Lynne (2012): Meeting the needs of translators in the age of e-lexicography: Exploring the possibilities. In: Granger, Sylviane/Paquot, Magali (Hrsg.): Electronic Lexicography. Oxford: Oxford University Press, 379-397.

Deppermann, Arnulf (2014): Handlungsverstehen und Intentionszuschreibung in der Interaktion I: Intentionsbekundungen mit wollen. In: Bergmann, Pia et al. (Hrsg.): Sprache im Gebrauch: räumlich, zeitlich, interaktional. Festschrift für Peter Auer. Heidelberg: Winter, 309-326.

Deppermann, Arnulf/Helmer, Henrike (2013): Standard des gesprochenen Deutsch: Begriff, methodische Zugänge und Phänomene aus interaktionslinguistischer Sicht. In: Hagemann, Jörg/Klein, Wolf Peter/Staffeldt, Sven (Hrsg.): Pragmatischer Standard. Tübingen: Stauffenburg, 111-141.

Deppermann, Arnulf/Proske, Nadine/Zeschel, Arne (2017) (Hrsg.): Verben im interaktiven Kontext. Bewegungsverben und mentale Verben im gesprochenen Deutsch. Tübingen: Narr.

Deppermann, Arnulf/Reineke, Silke (2017): Epistemische Praktiken und ihre feinen Unterschiede: Verwendungen von ich dachte in gesprochener Sprache. In: Deppermann, Arnulf/Proske, Nadine/Zeschel, Arne (Hrsg.): Verben im interaktiven Kontext. Bewegungsverben und mentale Verben im gesprochenen Deutsch. Tübingen: Narr, 337-375.

Domínguez Vázquez, María J./Mollica, Fabio/Nied Curcio, Martina (2014): Simplexverben im Italienischen und Spanischen vs. Präfix- und Partikelverben im Deutschen: Eine Untersuchung zum Gebrauch von Online-Wörterbüchern bei der Übersetzung. In: Domínguez Vázquez, María J./Mollica, Fabio/Nied Curcio, Martina (Hrsg.): Zweisprachige Lexikographie zwischen Translation und Didaktik. Berlin/New York: de Gruyter, 179-220.

Egido Vicente, María/Meliss, Meike (2017): ¿Cómo usar diccionarios bilingües en línea? Ejemplos para el desarrollo de las competencias lexicográficas en la enseñanza del alemán como lengua extranjera. In: RILE 7/2017, 31-53. [Unter: <https://revistes.urv.cat/index.php/rile/ article/view/1911>; letzter Zugriff: 16. 05. 2018].

Fernández Méndez, Manuel/Franco Barros, Mario/Sueiro Orallo, Irene (2016): El uso de los diccionarios de / para ELE y DaF: análisis de una encuesta desde una perspectiva contrastiva. In: Robles i Sabater, Ferran/Reimann, Daniel/Sánchez Prieto, Raúl (Hrsg.): Sprachdidaktik Spanisch-Deutsch. Forschungen an der Schnittstelle von Linguistik und Fremdsprachendidaktik. Tübingen: Narr, 73-92.

Flinz, Carolina (2014): Wörterbuchbenutzung: Ergebnisse einer Umfrage bei italienischen DaFLernern. In: Abel, Andrea/Vettori, Chiara/Ralli, Natascia (Hrsg.): Proceedings of the 16th EURALEX International Congress: The User in Focus. 15-19 July 2014. Bolzano/Bozen, Italy. Bolzano/Bozen: Institute for Specialised Communication and Multilingualism, 213-224. [Unter: <https://euralex.org/category/publications/euralex-2014/>; letzter Zugriff: 16. 05. 2018].

Günthner, Susanne (2017): Diskursmarker in der Interaktion Formen und Funktionen univerbierter guck mal- und weißt du-Konstruktionen. In: Blühdorn, Hardarik/Deppermann, Arnulf/Helmer, Henrike/Spranz-Fogasy, Thomas (Hrsg.): Diskursmarker im Deutschen. Reflexionen und Analysen. Göttingen: Verlag für Gesprächsforschung, 103-130.

Handwerker, Brigitte/Bäuerle, Rainer/Sieberg, Bernd (2016) (Hrsg.): Gesprochene Fremdsprache Deutsch. 1. Aufl. Baltmannsweiler: Schneider.

Hansen, Carsten/Hansen, Martin H. (2012): A dictionary of spoken Danish. In: Vatvedt Fjeld, Ruth/ Torjusen, Julie Matilde (Hrsg.): Proceedings of the 15th EURALEX International Congress. 7-11 August 2012. Oslo, Norway. Oslo: University of Oslo, 929-935. [Unter: <https:// euralex.org/category/publications/euralex-oslo-2012/>; letzter Zugriff: 16. 05. 2018). 
Imo, Wolfgang (2007): Construction Grammar und Gesprochene-Sprache-Forschung. Konstruktionen mit zehn matrixsatzfähigen Verben im gesprochenen Deutsch. Tübingen: Niemeyer.

Imo, Wolfgang (2013): Sprache in Interaktion. Analysemethoden und Untersuchungsfelder. Berlin/ Boston: de Gruyter.

Imo, Wolfgang/Moraldo, Sandro M. (2015) (Hrsg.): Interaktionale Sprache und ihre Didaktisierung im DaF-Unterricht. Tübingen: Stauffenburg.

Klosa, Annette/Koplenig, Alexander/Töpel, Antje (2011): Benutzerwünsche und Meinungen zu einer optimierten Wörterbuchpräsentation - Ergebnisse einer Onlinebefragung zu elexiko. Mannheim: Institut für Deutsche Sprache. [Unter: <http://pub.ids-mannheim.de/laufend/ opal/opal11-3.html>; letzter Zugriff: 16. 05. 2018].

Klosa, Annette/Tiberius, Carole (2016): Der lexikografische Prozess. In: Klosa, Annette/MüllerSpitzer, Carolin (Hrsg.): Internetlexikografie. Ein Kompendium. Berlin/Boston: de Gruyter, 65-110.

Kupietz, Marc/Schmidt, Thomas (2015): Schriftliche und mündliche Korpora am IDS als Grundlage für die empirische Forschung. In: Eichinger, Ludwig M. (Hrsg.): Sprachwissenschaft im Fokus. Positionsbestimmungen und Perspektiven. Berlin u. a.: de Gruyter, 297-322.

Lew, Robert (2015): Opportunities and limitations of user studies. In: Tiberius, Carole/MüllerSpitzer, Carolin (Hrsg.): Research into dictionary use/Wörterbuchbenutzungsforschung. 5. Arbeitsbericht des wissenschaftlichen Netzwerks „Internetlexikografie“. Mannheim: Institut für Deutsche Sprache, 6-16. [Unter: <http://pub.ids-mannheim.de/laufend/opal/opal152.html>; letzter Zugriff: 16. 05. 2018].

Lew, Robert/Adamska-Sałaciak, Arleta (2015): A case for bilingual learners' dictionaries. In: ELT Journal 69/1, 47-57.

Lew, Robert/de Schryver, Gilles-Maurice (2014): Dictionary users in the digital revolution. In: International Journal of Lexicography 27/4, 341-359.

Marello, Carla (2014): Using mobile bilingual dictionaries in an EFL class. In: Abel, Andrea/Vettori, Chiara/Ralli, Natascia (Hrsg.): Proceedings of the 16th EURALEX International Congress: The User in Focus. 15-19 July 2014. Bolzano/Bozen, Italy. Bolzano/Bozen: Institute for Specialised Communication and Multilingualism, 63-83. [Unter: <https://euralex.org/ category/publications/euralex-2014/>; letzter Zugriff: 16. 05. 2018].

Meliss, Meike (2015): Was suchen und finden Lerner des Deutschen als Fremdsprache in aktuellen Wörterbüchern? Auswertung einer Umfrage und Anforderungen an eine aktuelle DaF-Lernerlexikographie. In: Info DaF 42/4. München: iudicium, 401-432.

Meliss, Meike (2016): Gesprochene Sprache in DaF-Lernerwörterbüchern. In: Handwerker, Brigitte/Bäuerle, Rainer/Sieberg, Bernd (Hrsg.): Gesprochene Fremdsprache Deutsch. Baltmannsweiler: Schneider, 179-199.

Meliss, Meike/Möhrs, Christine (2017): Die Entwicklung einer lexikografischen Ressource im Rahmen des Projekts LeGeDe. In: Sprachreport 4/2017, 42-52. [Unter: <http://pub.idsmannheim.de/laufend/sprachreport/pdf/sr17-4.pdf>; letzter Zugriff: 16. 05. 2018].

Meliss, Meike/Möhrs, Christine (i. Dr.): Lexik in der spontanen, gesprochensprachlichen Interaktion: Eine anwendungsorientierte Annäherung aus der DaF-Perspektive. In: Journal GFL. [Unter: <http://www.gfl-journal.de/>; letzter Zugriff: 08.11.2018].

Meliss, Meike/Möhrs, Christine/Ribeiro Silveira, Maria (2018): Erwartungen an eine korpusbasierte lexikografische Ressource zur „Lexik des gesprochenen Deutsch in der Interaktion“: Ergebnisse aus zwei empirischen Studien. In: Zeitschrift für Angewandte Linguistik 68/1, 103-138.

Meliss, Meike/Möhrs, Christine/Batinić, Dolores/Perkuhn, Rainer (2018): Creating a List of Headwords for a Lexical Resource of Spoken German. In: Čibej, Jaka/Gorjanc, Vojko/Kosem, Iztok/Krek, Simon (Hrsg.): Proceedings of the XVIII EURALEX International Congress. 
Lexicography in Global Contexts, 17-21 July, Ljubljana. Ljubljana: Znanstvena založba Filozofske fakultete Univerze v Ljubljani, 1009-1016.

Möhrs, Christine/Meliss, Meike/Batinić, Dolores (2017): LeGeDe - Towards a corpus-based lexical resource of spoken German. In: Kosem, Iztok et al. (Hrsg.): Electronic Lexicography in the 21st Century. Proceedings of eLex 2017 conference. 19-21 September 2017. Leiden, the Netherlands. Brno: Lexical Computing CZ s.r.o, 281-298. [Unter: <https://elex.link/ elex2017/proceedings-download/>; letzter Zugriff: 16. 05. 2018].

Moon, Rosamund (1998): On using spoken data in corpus lexicography. In: Fontenelle, Thierry et al. (eds.): Proceedings of the 8th EURALEX International Congress. 4-8 August 1998. Liège, Belgium. Liège: University of Liège, 347-355.

Moraldo, Sandro M./Missaglia, Federica (2013) (Hrsg.): Gesprochene Sprache im DaF-Unterricht. Grundlagen - Ansätze - Praxis. 1. Aufl. Heidelberg: Winter.

Müller-Spitzer, Carolin (2014a): Methoden der Wörterbuchbenutzungsforschung. In: Lexicographica 30/1, 112-151.

Müller-Spitzer, Carolin (2014b) (Hrsg.): Using Online Dictionaries. Berlin/Boston: de Gruyter.

Müller-Spitzer, Carolin (2016a): Aufgaben und Relevanz der Wörterbuchbenutzungsforschung Mitte der 2010er Jahre. In: Schierholz, Stefan J. et al. (Hrsg.): Wörterbuchforschung und Lexikographie. Berlin/Boston: de Gruyter, 275-294.

Müller-Spitzer, Carolin (2016b): Wörterbuchbenutzungsforschung. In: Klosa, Annette/MüllerSpitzer, Carolin (Hrsg.): Internetlexikografie. Ein Kompendium. Berlin/Boston: de Gruyter, 291-342.

Müller-Spitzer, Carolin/Koplenig, Alexander/Töpel, Antje (2012): Online dictionary use: Key findings from an empirical research project. In: Granger, Sylviane/Paquot, Magali (Hrsg.): Electronic Lexicography. Oxford: Oxford University Press, 425-457.

Müller-Spitzer, Carolin/Koplenig, Alexander/Wolfer, Sascha (2018): Dictionary usage research in the Internet era. In: Fuertes Olivera, Pedro Antonio (Hrsg.): The Routledge Handbook of Lexicography. London: Routledge, 715-734.

Müller-Spitzer, Carolin/Wolfer, Sascha/Koplenig Alexander (2015): Observing online dictionary users: Studies using Wiktionary log files. In: International Journal of Lexicography 28/1, 1-26.

Nied Curcio, Martina (2014): Die Benutzung von Smartphones im Fremdsprachenerwerb und -unterricht. In: Abel, Andrea/Vettori, Chiara/Ralli, Natascia (Hrsg.): Proceedings of the 16th EURALEX International Congress: The User in Focus. 15-19 July 2014. Bolzano/Bozen, Italy. Bolzano/Bozen: Institute for Specialised Communication and Multilingualism, 213-224. [Unter: <https://euralex.org/category/publications/euralex-2014/>; letzter Zugriff: 16. 05. 2018].

Nied Curcio, Martina (2015): Wörterbuchbenutzung und Wortschatzerwerb. Werden im Zeitalter des Smartphones überhaupt noch Vokabeln gelernt? In: Info DaF 42/5. München: iudicium, 445-468.

Osterroth, Andreas (2018): „Antworte (nicht) im ganzen Satz!“. Gesprochener Standard in Lehrplänen und Bildungsstandards. In: Albert, Georg/Diao-Klaeger, Sabine (Hrsg.): Mündlicher Sprachgebrauch zwischen Normorientierung und pragmatischen Spielräumen. Tübingen: Stauffenburg, 117-131.

Perkuhn, Rainer/Keibel, Holger/Kupietz, Marc (2012): Korpuslinguistik. Paderborn: Fink.

Prensky, Marc (2001): Digital Natives, Digital Immigrants Part 1. In: On the Horizon 9/5, 1-6. [Unter: 〈https://doi.org/10.1108/10748120110424816>; letzter Zugriff: 16. 05. 2018].

Reeg, Ulrike/Gallo, Pasquale/Moraldo, Sandro M. (2012) (Hrsg.): Gesprochene Sprache im DaFUnterricht. Zur Theorie und Praxis eines Lerngegenstandes. Berlin: Waxmann.

Schmidt, Thomas (2014a): Gesprächskorpora und Gesprächsdatenbanken am Beispiel von FOLK und DGD. In: Gesprächsforschung - Online-Zeitschrift zur verbalen Interaktion 15, 196-233. [Unter: 〈http://www.gespraechsforschung-online.de/2014.html〉; letzter Zugriff: 16. 05. 2018]. Schmidt, Thomas (2014b): The research and teaching corpus of spoken German - FOLK. In: Calzolari, Nicoletta et al. (Hrsg.): Proceedings of the 9th International Conference on 
Language Resources and Evaluation (LREC'14). 26-31 May 2014. Reykjavik, Iceland. Iceland: ELRA, 383-387. [Unter: 〈http://www.lrec-conf.org/proceedings//rec2014/index.html>; letzter Zugriff: 16.05 .2018 ].

Schmidt, Thomas (2016): Good practices in the compilation of FOLK, the research and teaching corpus of spoken German. In: Kirk, John M./Andersen, Gisle (Hrsg.): Compilation, transcription, markup and annotation of spoken corpora. Special issue of International Journal of Corpus Linguistics 21/3, 396-418.

Schmidt, Thomas (2017): DGD - Die Datenbank für Gesprochenes Deutsch. Mündliche Korpora am Institut für Deutsche Sprache (IDS) in Mannheim. In: Zeitschrift für Germanistische Linguistik 45/3, 451-463.

Sieberg, Bernd (2013): Sprechen lehren, lernen und verstehen. Grammatik und Übungen zu Ausdrucksweisen und Strukturen mündlicher Kommunikation. Stufenübergreifendes Studien- und Übungsbuch für den DaF-Bereich. Tübingen: Groos.

Tarp, Sven (2009): Lexicography in the borderland between knowledge and non-knowledge. General lexicographical theory with particular focus on learner's lexicography. Tübingen: Niemeyer.

Tono, Yukio (2001): Research on dictionary use in the context of foreign language learning. Focus on reading comprehension. Tübingen: Niemeyer.

Töpel, Antje (2014): Review of research into the use of electronic dictionaries. In: Müller-Spitzer, Carolin (ed.): Using Online Dictionaries. Berlin/Boston: de Gruyter, 13-54.

Trap-Jensen, Lars (2004): Spoken language in dictionaries: Does it really matter? In: Williams, Geoffrey/Vessier, Sandra (Hrsg.): Proceedings of the 11th EURALEX International Congress. 6-10 July 2004. Lorient, France. Lorient: Université de Bretagne Sud, 311-318.

Trim, John/North, Brian/Coste, Daniel (2001): Gemeinsamer europäischer Referenzrahmen für Sprachen: lernen, lehren, beurteilen. Niveau A1, A2, B1, B2, C1, C2. Berlin: Langenscheidt.

Verdonik, Darinka/Sepesy Maučec, Mirjam (2017): A speech corpus as a source of lexical information. In: International Journal of Lexicography 30/2, 143-166.

Welker, Herbert A. (2010): Dictionary use: A general survey of empirical studies. Brasilia: Eigenverlag.

Welker, Herbert A. (2013): Methods in research of dictionary use. In: Gouws, Rufus H. et al. (Hrsg.): Dictionaries. An International Encyclopedia of Lexicography. Supplementary Volume: Recent Developments with Focus on Electronic and Computational Lexicography. Berlin/ Boston: de Gruyter, 540-547.

Wiegand, Herbert E. (1998): Wörterbuchforschung. Untersuchungen zur Wörterbuchbenutzung, zur Theorie, Geschichte, Kritik und Automatisierung der Lexikographie. Berlin/New York: de Gruyter.

Wolfer, Sascha/Koplenig, Alexander/Meyer, Peter/Müller-Spitzer, Carolin (2014): Dictionary users do look up frequent and socially relevant words. Two log-file analyses. In: Abel, Andrea/ Vettori, Chiara/Ralli, Natascia (Hrsg.): Proceedings of the 16th EURALEX International Congress: The User in Focus. 15-19 July 2014. Bolzano/Bozen, Italy. Bolzano/Bozen: Institute for Specialised Communication and Multilingualism, 281-290. [Unter: <https://euralex.org/ category/publications/euralex-2014/>; letzter Zugriff: 16. 05. 2018].

Zöfgen, Ekkehard (2010): Wörterbuchdidaktik. In: Hallet, Wolfgang/Königs, Frank G. (Hrsg.): Handbuch Fremdsprachendidaktik. Seelze: Kallmeyer, 107-110.

\subsection{Ressourcen und Online-Wörterbücher}

DEREKo = Deutsches Referenzkorpus/Archiv der Korpora geschriebener Gegenwartssprache 2017-I (Release vom 08. 03. 2017). Mannheim: Institut für Deutsche Sprache. [Unter: 
<http://www.ids-mannheim.de/direktion/kl/projekte/korpora/releases.html>; letzter Zugriff: 16. 05. 2018].

Duden-online. [Unter: 〈https://www.duden.de/>; letzter Zugriff: 16. 05. 2018].

DWDS = Das Wortauskunftssystem zur deutschen Sprache in Geschichte und Gegenwart. [Unter: <www.dwds.de.>; letzter Zugriff: 16. 05. 2018].

elexiko = Online-Wörterbuch zur deutschen Gegenwartssprache. [Unter: 〈http://www.owid.de/wb/ elexiko/start.html>; letzter Zugriff: 08.11. 2018].

FOLK = Forschungs- und Lehrkorpus Gesprochenes Deutsch (Release 2.10, Stand: Mai 2018).

Mannheim: Institut für Deutsche Sprache. [Unter: 〈http://agd.ids-mannheim.de/folk.shtml>; letzter Zugriff: 08.11.2018].

Linguee. [Unter: 〈https://www.linguee.de/>; letzter Zugriff: 16. 05. 2018].

LEO. [Unter: <https://www.leo.org/>; letzter Zugriff: 16. 05. 2018].

Ordbog over Dansk Talesprog. [Unter: 〈http://odt.hum.ku.dk/>; letzter Zugriff: 16. 05. 2018]

OWID = Online-Wortschatz-Informationssystem Deutsch (2008 ff.). Mannheim: Institut für

Deutsche Sprache. [Unter: 〈www.owid.de〉; letzter Zugriff: 16. 05. 2018].

Pons Online-Wörterbuch. [Unter: <https://de.pons.com/>; letzter Zugriff: 16. 05. 2018]. 